\title{
Ocean Engineering
}

\section{Turbine design dependency to turbulence: An experimental study of three scaled tidal turbines}

\author{
Myriam Slama a , Grégory Pinon ${ }^{\text {a,* }}$, Charifa El Hadi a, Michael Togneri ${ }^{\mathrm{b}}$, Benoît Gaurier ${ }^{\mathrm{c}}$, \\ Grégory Germain ${ }^{c}$, Jean-Valéry Facq ${ }^{c}$, José Nuño ${ }^{d}$, Pablo Mansilla ${ }^{d}$, Erwann Nicolas ${ }^{e}$, \\ Julie Marcille ${ }^{\mathrm{e}}$, André Pacheco ${ }^{\mathrm{f}}$ \\ ${ }^{a}$ Normandie Univ, UNILEHAVRE, UMR 6294 CNRS, LOMC 76600 Le Havre, France \\ ${ }^{\mathrm{b}}$ Energy \& Environment Research Group, Swansea University, Bay Campus, Swansea SA1 8EN, UK \\ ${ }^{\mathrm{c}}$ IFREMER, 150 quai Gambetta, BP 699, 62321 Boulogne-sur-mer, France \\ ${ }^{\mathrm{d}}$ Magallanes Renovables, Prego de Montaos 7, Redondela, 36800, Spain \\ e Sabella S.A.S., 11 Rue Félix Le Dantec, 29000 Quimper, France \\ ${ }^{\mathrm{f}}$ University of Algarve, Edificio 7, Campus de Gambelas, 8005-139 Faro, Portugal
}

\section{A R T I C L E I N F O}

\section{Keywords:}

Marine current turbine

Performance

Turbulence

Tank testing

Coherence

Spectral analysis

\begin{abstract}
A B S T R A C T
In this paper the turbulence effects are studied for three rotors mounted on the same instrumented hub. Two scaled models of industrial turbines and one open-geometry turbine are considered. The turbulence characteristics are obtained from 2D Laser Doppler Velocimeter measurements and the turbine behaviour is analysed from thrust and torque measurements. Three turbulence intensities and a large range of tip speed ratios and flow velocities are considered. The results are anonymised in order to ensure confidentiality. The rotors have different blade profiles, blade numbers and solidity. The rotor design largely modifies the mean power and thrust coefficients. The turbulence intensity only slightly changes these results but has a larger influence on the fluctuating loads than the different rotor designs. The spectral analysis of the rotor torque and thrust shows that, at low frequencies the load variations are correlated to those of the flow velocity with some differences due to the turbulence intensity levels. The coherences between the loads and the velocity seem to be not affected by the rotor type. At high frequencies, the load variations are correlated to the speed control unit of the scaled model and the rotor design has an impact on the rotational speed and loads coherences.
\end{abstract}

\section{Introduction}

Tidal energy constitutes an interesting resource for European countries in the Atlantic area and several concepts were proposed during the last decades. However, the development of this sector is still limited due to uncertainty in the engineering design. Different strategies exist to design tidal turbines and particularly their blades which are critical components (Liu and Veitch, 2012). The optimisation of the turbine performance using a hydraulic design allows to maximise the energy output and decrease the loads experienced by the supporting structure and other components (Kaufmann et al., 2019). The blade robustness can also be improved with the selection of an appropriate material. For example, in the case of turbines with fixed pitch blades, composite blades can be designed to passively adapt their angle of attack during operation and hence reduce the loads (Murray et al., 2018).

One of the issues that should be taken into account for blade design is the load variations due to the ambient turbulence of the deployment site, which affect the reliability of the turbine. Several in situ velocity measurements have shown that the ambient turbulence intensity may vary significantly depending on the implementation site. The turbulence intensity measured goes from $\approx 4-9 \%$ in Strangford Lough (UK) (MacEnri et al., 2013), to $\approx 10-13 \%$ in Puget Sound (USA) (Thomson et al., 2012; McCaffrey et al., 2015) and Sound of Islay (UK) (Milne et al., 2013), or to $\approx 20-30 \%$ in East River (USA) (Li et al., 2010). Moreover, recent studies (Mycek et al., 2014; Blackmore et al., 2016) have shown that the ambient turbulence has an important influence on the turbine performance. Particularly, Mycek et al. (2014) found that the fluctuations of the power and thrust coefficients increase drastically with the turbulence intensity. Thus a high turbulence intensity has an impact on the fatigue of the turbine.

In this context, the MONITOR project (Togneri et al., 2019), supported by the Interreg Atlantic Area programme, was launched in 2018. Its global objectives are to investigate, using multiple testing

\footnotetext{
* Corresponding author.

E-mail address: gregory.pinon@univ-lehavre.fr (G. Pinon).
} 


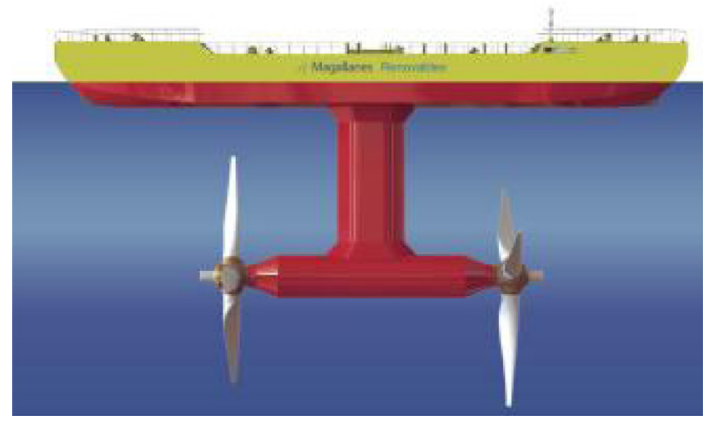

Fig. 1. Schematic CAD representation of Magallanes Renovables' ATIR tidal platform.

methodologies (numerical, laboratory and at-sea), the reliability of tidal energy converters (TEC) subjected to real in-situ flow conditions (possibly with high ambient turbulence (Mycek et al., 2014; Blackmore et al., 2016; Milne et al., 2016; Togneri et al., 2017) and severe wave conditions (Faudot and Dahlhaug, 2012; Luznik et al., 2013; Guo et al., 2018)) and to develop tools to help TEC developers improve device reliability (Scheijgrond et al., 2019).

The aim of this study is to characterise the fluctuating loads for a single turbine immersed in a regular current flow with different levels of turbulence. The turbine scaled models used during the trials are based on two pre-commercial prototypes, namely Magallanes Renovables' ATIR tidal platform and the bottom mounted D12 turbine of Sabella. The ATIR (Fig. 1) is a floating device composed of a 3bladed bi-rotor turbine with variable pitch mechanisms to optimise the energy production. The turbine diameter is $D=19 \mathrm{~m}$, its overall length is $45 \mathrm{~m}$, its breadth is $6 \mathrm{~m}$ and its weight is 350 tons. The device is designed for a maximum power output of $2 \mathrm{MW}$. Its platform is anchored to the sea bottom by four mooring lines, to the bow and stern. A first full scale prototype was tested at the Ría de Vigo in Spain and generated power for current velocities from $1 \mathrm{~m} / \mathrm{s}$ to $3 \mathrm{~m} / \mathrm{s}$ (DiazDorado et al., 2021). The platform was then deployed at the European Marine Energy Centre (EMEC) in Scotland, during summer 2018. The device is reaching a TRL (Technology Readiness Level) of 9. Sabella's D12 (Fig. 2) is composed of a single rotor with 5 fixed (no-pitch) and symmetrical blades. The turbine diameter is $12 \mathrm{~m}$ for a power output of $500 \mathrm{~kW}$. The D12 is scheduled for installation in 2021; Sabella's earlier D10 model operating on the same design principles has already undergone two test deployments in 2015 and 2018.

Finally, results from the open-geometry scaled turbine of the IFREMER (Gaurier et al., 2015, 2019, 2020) will also be used as a matter of comparison. Therefore, there is a total of three turbines at similar model scales that were tested for the same inflow conditions with the same test facility and instrumentation. Each turbine has a different blade design: IFREMER's turbine is not optimised for one site but is designed to operate for a large range of operating velocities; the ATIR blades are designed for a target operating velocity; the D12 orientation is fixed and its blade profile is symmetric in order to work in bi-directional flow.

This work is divided in four main sections, following this introduction. First, the experimental set-up and the upstream flow characteristics are described in Sections 2 and 3. Then the results obtained for the time averaged torque and thrust coefficients and their fluctuations, for each turbine, are presented in Section 4. Lastly, a spectral analysis of the rotor loads is carried out in Section 5 .

\section{Experimental set-up}

In this section, the turbine models as well as the flume tank and the instrumentation are described. The different flow conditions considered are also presented.

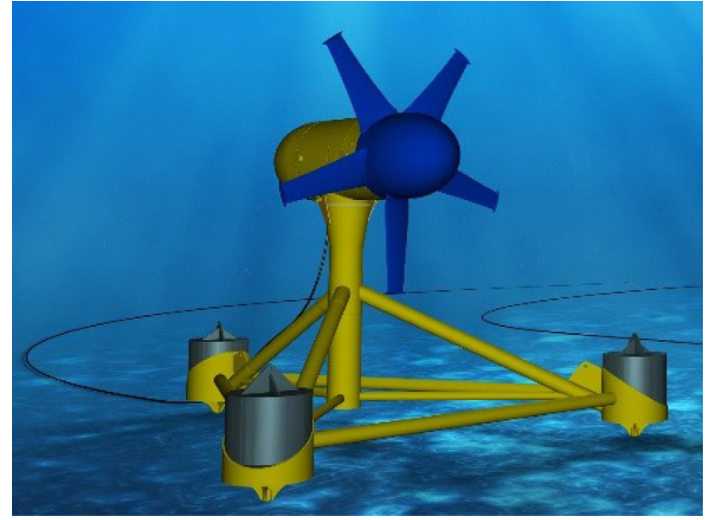

Fig. 2. Representation of Sabella's D12 turbine.

\subsection{Flume tank description and experimental configurations}

The trials were carried out in the IFREMER wave and current flume tank in Boulogne-sur-mer, presented in Fig. 3. The flume tank working section is $18 \mathrm{~m}$ long, $4 \mathrm{~m}$ wide and $2 \mathrm{~m}$ deep. The streamwise flow velocity ranges from 0.1 to $2.2 \mathrm{~m} / \mathrm{s}$.

The experimental set-up, illustrated in Figs. 4 and 5, was the same for both trial campaigns. The hub of the scaled turbine was set at a height of $1 \mathrm{~m}$ below the free surface. The flow conditions were monitored at the hub height and at $1.4 \mathrm{~m}$ (approximately 2 times the turbine diameter) upstream of the turbine with a bi-dimensional Laser Doppler Velocimeter (LDV). This system is composed of four laser beams with two wavelengths: $488 \mathrm{~nm}$ and $514.5 \mathrm{~nm}$. The measurement volume, created by the intersection of the laser beams, is approximately $0.01 \mathrm{~mm}^{3}$ (the measurement volume is $2.51 \mathrm{~mm}$ long and the laser beams thickness is approximately $0.12 \mathrm{~mm}$ ). The LDV estimates the velocity components of the particles passing through this volume. The particles used in the IFREMER flume tank are silver-coated glass spheres with a diameter of $10 \mu \mathrm{m}$. Since a measurement value corresponds to a detected particle, the sampling frequency is irregular. For this study, the mean sampling rate is between $200 \mathrm{~Hz}$ and $500 \mathrm{~Hz}$. An Acoustic Doppler Velocimeter (ADV) was also placed in the flume tank but it was not used for the present study. More details about the flume tank and the instrumentation can be found in Gaurier et al. (2018, 2019).

\subsection{Upstream flow conditions}

The ambient turbulence intensity $I_{\infty}$ generated in the flume tank can be regulated using flow straighteners placed at the inlet of the working section. It goes from $I_{\infty} \approx 1.5 \%$ when both a grid and a honeycomb (see Figs. 4 and 6 ) are used, to $I_{\infty} \approx 3 \%$ when only the honeycomb is used and to a higher value of $I_{\infty} \approx 15 \%$ when removing all the flow straighteners. Thus, this $I_{\infty}$ range corresponds to measured in-situ values (Mycek et al., 2014; Milne et al., 2013; Thomson et al., 2012; Togneri et al., 2017).

In the present study, the three possible turbulence intensities were used. The different cases will be referred to as Low Turbulence Intensity (LTI), Medium Turbulence Intensity (MTI) and High Turbulence Intensity (HTI) in the following sections.

Lastly, the mean upstream axial velocity range used in these trials goes from $U_{\infty}=0.80 \mathrm{~m} / \mathrm{s}$ to $U_{\infty}=1.4 \mathrm{~m} / \mathrm{s}$.

\subsection{Turbine models description}

Both the ATIR and the D12 scaled models, shown in Fig. 7, are based on the existing IFREMER generic turbine, a tri-bladed turbine 

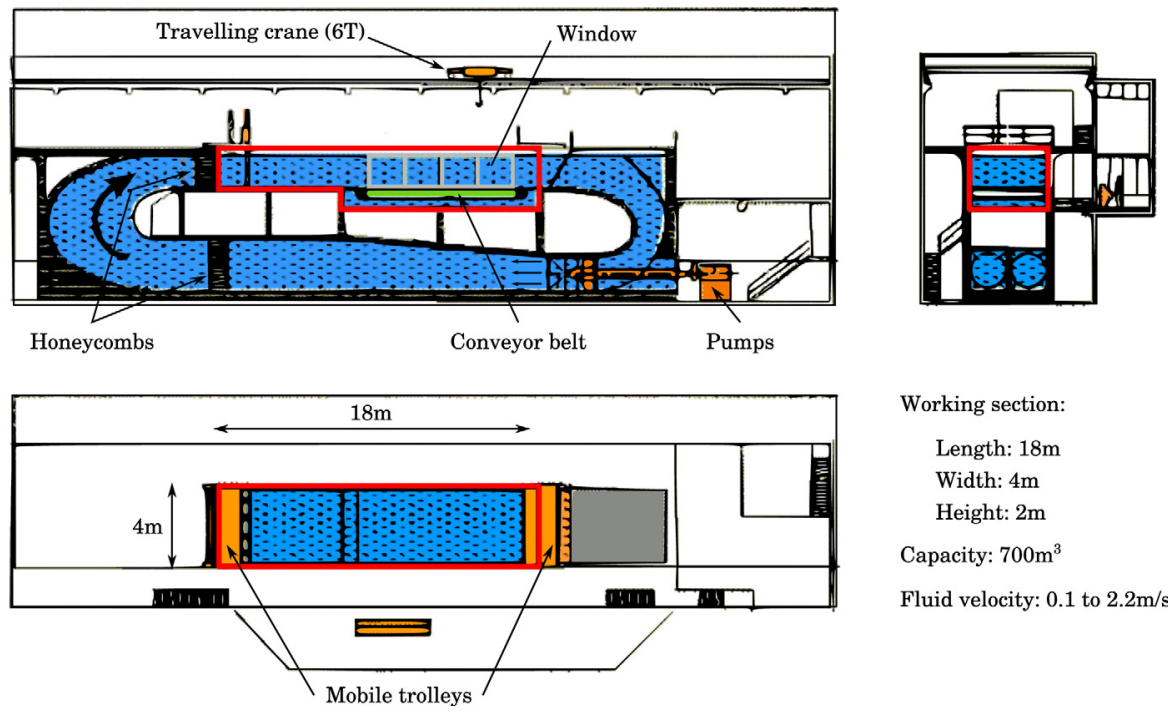

Working section:

Length: $18 \mathrm{~m}$

Width: $4 \mathrm{~m}$

Height: $2 \mathrm{~m}$

Capacity: $700 \mathrm{~m}^{3}$

Fluid velocity: 0.1 to $2.2 \mathrm{~m} / \mathrm{s}$

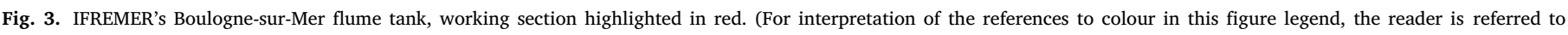
the web version of this article.)

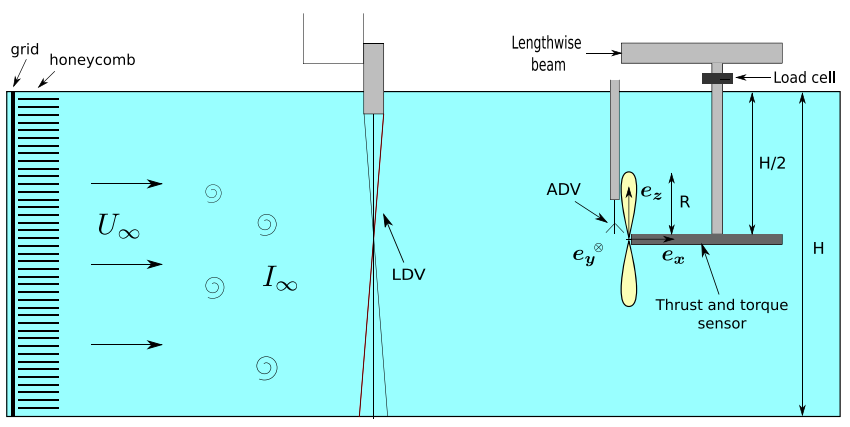

Fig. 4. Schematic side view of the test configuration.

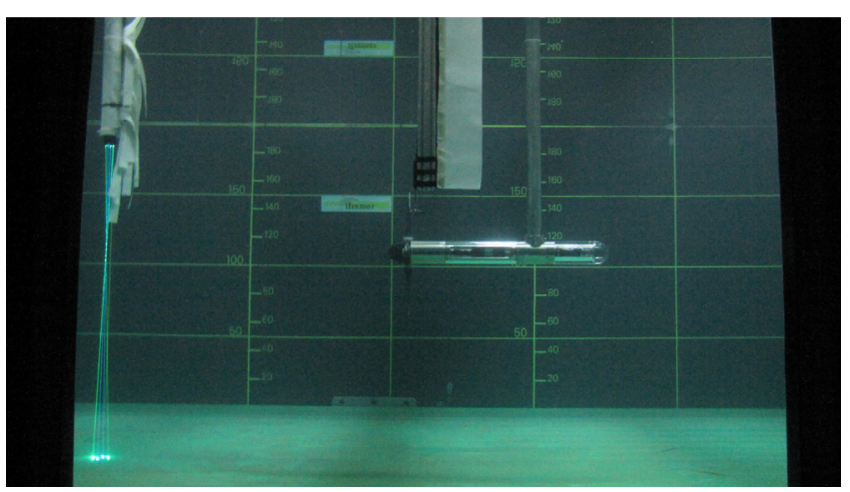

Fig. 5. Side view of the turbine in the flume tank with the 2D LDV (left) and the ADV systems.

with a horizontal axis, used in previous works (Gaurier et al., 2019, 2020). Only the blades and the hub were changed to adapt the turbine to the industrial device geometries. For the D12, a nacelle diameter extension was added to better represent this particular characteristics of the device. Finally for the ATIR, only one of the rotors was modelled. Model parameters, such as the hub radius and the blade lengths, are detailed in Table 1 . The blades of the IFREMER turbine are designed from a NACA 63418 profile. Magallanes Renovables and Sabella blade

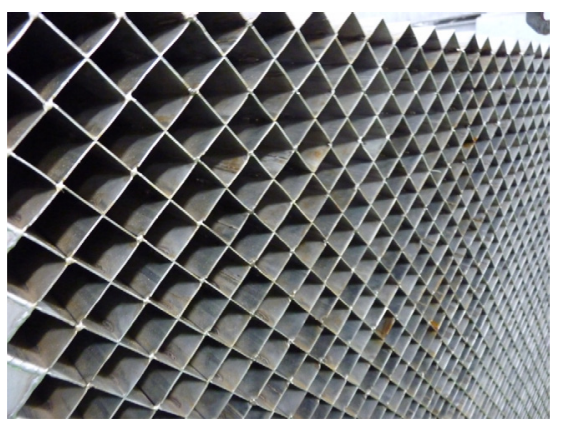

Fig. 6. Picture of the honeycomb flow straightener.

Table 1

Turbine model parameter description.

\begin{tabular}{llll}
\hline Description & IFREMER & ATIR & D12 \\
\hline Profile & NACA 63418 & - & - \\
Rotor radius $R[\mathrm{~mm}]$ & 362 & 338 & 300 \\
Hub radius [mm] & 55 & 55 & 96 \\
Hub length [mm] & 720 & 720 & 720 \\
Number of blades & 3 & 3 & 5 \\
Blade length [mm] & 307 & 283 & 204 \\
Direction of rotation & CC & C & CC \\
Blade material & CF & A & CF \\
\hline
\end{tabular}

C: clockwise; CC: counter-clockwise; CF: Carbon fibre; A: Aluminium.

profiles are confidential. Both Sabella and IFREMER blades are made of carbon fibre. They were produced by the same manufacturer and with the same technique. The blades of the ATIR scaled model are made of aluminium.

The scale of the ATIR and the D12 models are 1:28 and 1:20 respectively. Considering the characteristics of the experimental set-up, the Froude criterion was preferred to the Reynolds criterion. Indeed, the full scale Reynolds numbers are around $10^{7}$ and these values cannot be obtained in the flume tank. The Froude scaling parameters chosen for both turbines are presented in Table 2. The Froude number is defined as:

$F r_{\infty}=\frac{U_{\infty}}{\sqrt{g \times H}}$ 
Table 2

Froude scaling characteristic between the prototype scale and the model scale for each turbine..

\begin{tabular}{lllllll}
\hline $\begin{array}{l}\text { Turbine physical } \\
\text { parameters }\end{array}$ & $\begin{array}{l}\text { Froude } \\
\text { scaling }\end{array}$ & $\begin{array}{l}\text { ATIR } \\
\text { scale } 1: 1\end{array}$ & $\begin{array}{l}\text { ATIR } \\
\text { scale } 1: 28\end{array}$ & $\begin{array}{l}\text { D12 } \\
\text { scale } 1: 1\end{array}$ & $\begin{array}{l}\text { D12 } \\
\text { scale } 1: 20\end{array}$ & $\begin{array}{l}\text { IFREMER } \\
-\end{array}$ \\
\hline Radius $R[\mathrm{~m}]$ & $1: \lambda$ & 9.5 & 0.338 & 6 & 0.300 & 0.362 \\
Water depth $H[\mathrm{~m}]$ & $1: \lambda$ & $56^{\mathrm{a}}$ & 2 & $40^{\mathrm{a}}$ & 2 & 2 \\
Flow speed $[\mathrm{m} / \mathrm{s}]$ & $1: \sqrt{\lambda}$ & {$[4.2-7.4]$} & {$[0.8-1.4]$} & {$[3.6-6.3]$} & {$[0.8-1.4]$} & {$[0.8-1.2]$} \\
TSR range $[-]$ & - & {$[0-8]$} & {$[0-8]$} & {$[0-6]$} & {$[0-6]$} & {$[0-8]$} \\
$R e_{\infty} \times 10^{5}[-]$ & $1: \lambda \sqrt{\lambda}$ & {$[400-700]$} & {$[2.7-4.7]$} & {$[220-380]$} & {$[2.4-4.2]$} & {$[2.9-4.3]$} \\
\hline
\end{tabular}

The chosen Froude scaling range is $F r_{\infty}=[0.18-0.32]$ for the three scaled turbines depending on the considered velocity.

${ }^{a}$ Hypothetical water depth based on the scale factor.
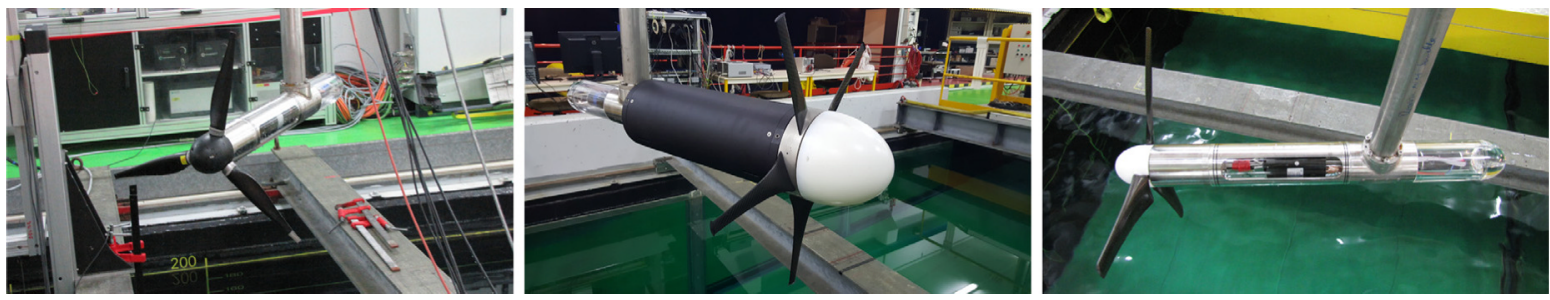

Fig. 7. Pictures of the scaled models: left is Magallanes Renovables' ATIR, middle is Sabella's D12, and right is IFREMER's generic turbine.

where $g=9.81 \mathrm{~m} / \mathrm{s}^{2}$ is the gravitational acceleration and $H$ is the water depth. The corresponding Reynolds numbers, defined as:

$R e_{\infty}=\frac{U_{\infty} \times R}{v}$

(where $v$ is the kinematic viscosity of the fluid), are also given in Table 2 as a matter of information.

The upstream velocity range used in this study $\left(U_{\infty}=0.80-1.4 \mathrm{~m} / \mathrm{s}\right)$ corresponds to a full scale velocity range of $4.2-7.4 \mathrm{~m} / \mathrm{s}$ for the ATIR and 3.6-6.3 m/s for the D12 turbine (see Table 2). In energetic sites (e.g Alderney race, Fromveur strait, Fall of Warness, Bay of Fundy, etc.), velocities at the order of $5 \mathrm{~m} / \mathrm{s}$ are regularly measured. Even higher velocities could be encountered for the highest tidal coefficients, which are not so frequent. Therefore, the velocity range studied here is relevant for the higher velocities encountered in highly energetic sites.

The blockage ratio $\alpha$ is defined as the ratio between the rotor cross-section area $S=\pi \times R^{2}$ and the flume tank transverse area $A=l \times H$ :

$\alpha=\frac{S}{A}=\frac{\pi \times R^{2}}{l \times H}$.

The blockage ratios for these trials are $\alpha \approx 4.5 \%$ and $\alpha \approx 3.5 \%$ for Magallanes Renovables and Sabella scaled turbines respectively. For the IFREMER model, this ratio is about $5.1 \%$. Since these ratios are relatively small and close, no blockage correction (such as Bahaj et al. (2007)) is applied.

The rotor of the scaled models is connected to a motor-gearbox assembly consisting of a gearbox, a DC motor, a ballast load and a motor speed control unit (Mycek et al., 2014; Gaurier et al., 2020), providing an active rotor speed control. The pitch of the blades is fixed in each case. For the ATIR turbine, the main difference between the model and the full-scale device, apart from size, is that the model has fixed pitch blades and the full scale model has variable pitch blades. This means that the blades of the model may not be at the optimum pitch angle in all operating conditions.

The Tip Speed Ratio (TSR) is defined as the ratio of the tip velocity of the blade to the upstream flow velocity:

$T S R=\frac{\omega_{x} \times R}{U_{\infty}}$

where $\omega_{x}$ is the axial rotation speed. In this study, the TSR varies from 0 to 8 for the ATIR model and from 0 to 6 for the D12 turbine.

The torque and thrust are directly measured on the rotation axis with a waterproof transducer. The latter is placed upstream of the rotor seals to prevent measuring friction effects. For the 3-bladed models

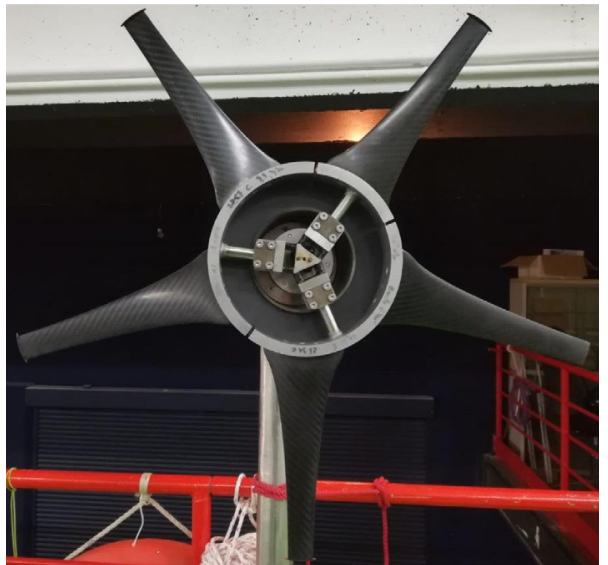

Fig. 8. Picture of the blade root load cells and the three blade supports of Sabella's D12 scaled model.

(IFREMER and ATIR), each blade root is equipped with a load-cell measuring two forces and three moments (see Gaurier et al. (2019) for more details). For the 5-bladed D12, three blade supports were manufactured in order to adapt the model to the IFREMER device. One of them was used to support only one blade and two blades were fixed on each of the other supports (see Fig. 8). Therefore, for the Sabella scaled turbine, the blade root loads were studied only for one blade.

Finally, during both trial campaigns, the turbine parameters and the flow velocity were monitored synchronously. The signals were sampled at a frequency of $120 \mathrm{~Hz}$, except for the LDV which has an irregular sampling rate. The acquisition time was set to $180 \mathrm{~s}$ for the LTI and MTI cases and to $360 \mathrm{~s}$ for the HTI.

\section{Upstream flow characterisation}

\subsection{Turbulence intensity}

The upstream turbulence intensity $I_{\infty}$ is defined as:

$I_{\infty}=100 \sqrt{\frac{1 / 3\left(\sigma_{u_{\infty}}^{2}+\sigma_{v_{\infty}}^{2}+\sigma_{w_{\infty}}^{2}\right)}{\bar{u}_{\infty}^{2}+\bar{v}_{\infty}^{2}+\bar{w}_{\infty}^{2}}}$

where $\sigma_{u_{\infty}}, \sigma_{v_{\infty}}$ and $\sigma_{w_{\infty}}$ are the standard deviations of the velocity components $u_{\infty}, v_{\infty}$ and $w_{\infty}$ of the upstream velocity $\boldsymbol{u}_{\infty}\left(\boldsymbol{u}_{\infty}=u_{\infty} \boldsymbol{e}_{\boldsymbol{x}}+\right.$ 
$v_{\infty} \boldsymbol{e}_{\boldsymbol{y}}+w_{\infty} \boldsymbol{e}_{z}$ ); refer to Fig. 4 for a definition of the unit vectors. The overbar denotes the time average. The mean streamwise velocity $\bar{u}_{\infty}$ is also denoted by $U_{\infty}$ in this paper.

In this study, the upstream flow velocity was measured with the LDV in the $x-y$ plane. Thus a bi-dimensional turbulence intensity is also defined:

$I_{\infty}^{2 D}=100 \sqrt{\frac{1 / 2\left(\sigma_{u_{\infty}}^{2}+\sigma_{v_{\infty}}^{2}\right)}{\bar{u}_{\infty}^{2}+\bar{v}_{\infty}^{2}}}$.

Table 3 presents the values obtained for the three turbulence intensity configurations (LTI, MTI and HTI), at $U_{\infty}=1.2 \mathrm{~m} / \mathrm{s}$, for the ATIR and D12 trial campaigns. The values for the HTI configuration for the IFREMER turbine come from another trial campaign (Gaurier et al., 2019, 2020), for which no flow measurement with a turbine was performed for the LTI and MTI conditions. The different statistics were estimated by considering the LDV measurements for all the TSR cases. The tri-dimensional values were calculated by assuming that $\sigma_{v_{\infty}}=\sigma_{w_{\infty}}$ (already validated from previous studies). As the onedimensional turbulence intensity, $I_{\infty}^{1 D}=\sigma_{u_{\infty}} / U_{\infty}$, is used in some studies (e.g. McCaffrey et al., 2015; Blackmore et al., 2016; Medina et al., 2017), these results are also given as a matter of information. Independently from the aforementioned campaigns, Table 3 also shows the turbulence statistics obtained from LDV measurements performed at the turbine hub position, without the scaled turbine, for $30 \mathrm{~min}$, for the LTI and HTI conditions as a matter of further comparison.

For the LTI case, the standard deviations of streamvise velocity, and thus the turbulence intensities, are slightly lower for the measurements carried out without the turbine. Aside from that, the results are very similar for the different configurations. Contrary to the lower turbulence intensity cases, the flow is almost isotropic for the HTI condition with $\sigma_{v_{\infty}} / \sigma_{u_{\infty}} \approx 1.1$. This is due to the honeycomb (see Fig. 6), placed in the flume tank for the low $I_{\infty}$ configurations, which constrains the flow in both the normal and transverse directions (Medina et al., 2017).

\subsection{Flow properties}

As the turbulence intensity does not provide any information about the turbulence spatial structure, some additional characteristics of the flow are also studied. Since the sampling rate of the velocity measurements is irregular, the LDV data are interpolated with a regular time sampling for the estimation of the spectra and the correlations presented in this section.

According to Blackmore et al. (2016), the integral length scale, which characterise the size of the most energetic turbulent eddies, is an important parameter. Assuming that the flow is statistically stationary, this length can be estimated from the autocorrelation function of the streamwise velocity:

$R(\tau)=\frac{\overline{u_{\infty}^{\prime}(t) u_{\infty}^{\prime}(t+\tau)}}{\sigma_{u_{\infty}}^{2}}$

where $u_{\infty}^{\prime}=u_{\infty}-U_{\infty}$ and $\tau$ is the time lag. The integral time scale $T$ is obtained by integrating the autocorrelation function with respect to time between $\tau=0$ and the first time lag for which $R=0$, denoted by $\tau_{0}$.

$T=\int_{0}^{\tau_{0}} R(\tau) \mathrm{d} \tau$.

Lastly, the integral length scale $L$ is estimated from $T$ using Taylor's hypothesis: $L=T U_{\infty}$.

The length scales estimated for the IFREMER (only for the HTI configuration), ATIR and D12 trial campaigns (at $U_{\infty}=1.2 \mathrm{~m} / \mathrm{s}$, considering all the TSR cases) as well as the measurements carried out without turbine are presented in Table 4. It can be observed that, for the four configurations, for the LTI and HTI cases, the integral length scale is $\approx 0.5-0.6 \mathrm{~m}$. For the MTI, $L$ is approximately twice the size.

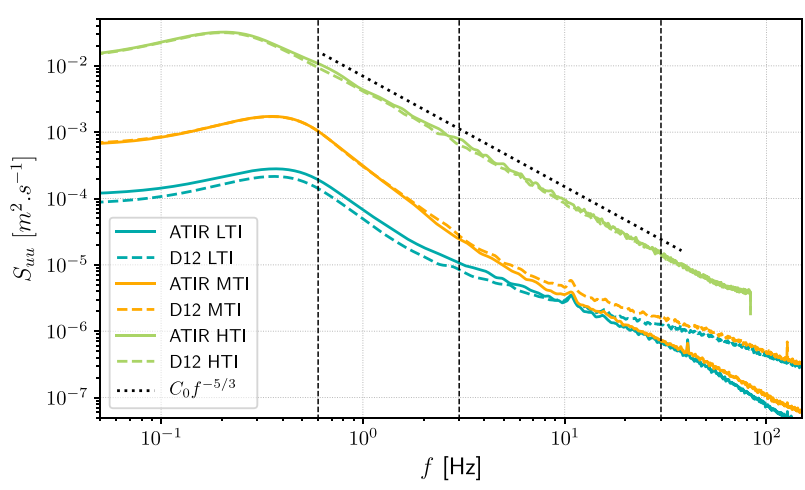

Fig. 9. Power spectral densities of the streamwise flow velocity, obtained at $U_{\infty}=$ $1.2 \mathrm{~m} / \mathrm{s}$ for the three turbulence intensities.

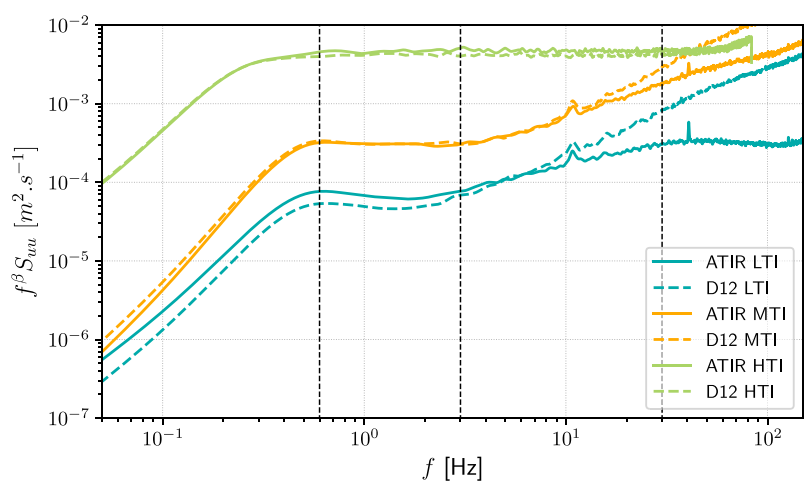

Fig. 10. Compensated spectra of the streamwise flow velocity, obtained at $U_{\infty}=$ $1.2 \mathrm{~m} / \mathrm{s}$ for the three turbulence intensities.

In the same way as Medina et al. (2017), the flow properties are also characterised via a spectral analysis of the upstream velocity. The power spectral densities (PSD) of the streamwise velocity, $S_{u u}$, presented in Fig. 9, were obtained using the scipy.signal.welch function of Python. For each turbulence intensity configuration, an inertial subrange is visible but the frequency range is narrower for the lowest values of $I_{\infty}: f \in[0.6 ; 2.5] \mathrm{Hz}$ for LTI, $f \in[0.6 ; 3] \mathrm{Hz}$ for MTI and $f \in[0.6 ; 30] \mathrm{Hz}$ for HTI. These ranges are more visible in Fig. 10 which presents the compensated velocity PSD $f^{\beta} S_{u u}$, where $\beta$ is the negative of the estimated slope.

The dissipation rate of the turbulent kinetic energy $\varepsilon$ can be estimated from $S_{\text {ии }}$ in the inertial subrange using Kolmogorov's $-5 / 3$ law (Pope, 2000):

$S_{\text {uu }}(k)=C \varepsilon^{2 / 3} k^{-5 / 3}$

where $C$ is the Kolmogorov constant $(C \approx 1.5)$ and $k$ is the wavenumber. Using Taylor's hypothesis (Pope, 2000), the wavenumber $k$ can be related to the frequency $f$ :

$k=\frac{2 \pi f}{U_{\infty}}$.

Then, the dissipation rate can be estimated from the following relation (Medina et al., 2017):

$\varepsilon=\left(\frac{C_{0}}{C}\right)^{3 / 2}\left(\frac{2 \pi}{U_{\infty}}\right)^{5 / 2}$

where $C_{0}$ is obtained from the velocity PSD such that $C_{0} f^{-\beta}$ fits $S_{u u}(f)$ in the inertial subrange. $C_{0}$ and the slope $\beta$ are estimated using a linear least-squares regression (see Table 4).

As can be seen in Fig. 9, except for the HTI case, in the inertial subrange, the PSD differ from Kolmogorov's law. The slopes estimated are larger than $5 / 3$ (see Table 4), particularly for the MTI. This result 
Table 3

Turbulence statistics measured during the trial campaigns for $U_{\infty}=1.2 \mathrm{~m} / \mathrm{s}$.

\begin{tabular}{|c|c|c|c|c|c|c|c|c|c|}
\hline Turbine & Case & $U_{\infty}[\mathrm{m} / \mathrm{s}]$ & $\sigma_{u_{\infty}}[\mathrm{m} / \mathrm{s}]$ & $\sigma_{v_{\infty}}[\mathrm{m} / \mathrm{s}]$ & $I_{\infty}^{1 D}[\%]$ & $\begin{array}{ll}I_{\infty}^{2 D}[\%] \\
\end{array}$ & $I_{\infty}^{3 D}[\%]$ & $\sigma_{v_{\infty}} / \sigma_{u_{\infty}}$ & Time $[\mathrm{s}]$ \\
\hline \multirow{2}{*}{ - } & LTI & 1.20 & 0.0202 & 0.0114 & 1.68 & 1.37 & 1.25 & 0.567 & 1800 \\
\hline & HTI & 1.17 & 0.158 & 0.175 & 13.6 & 14.3 & 14.5 & 1.10 & 1800 \\
\hline \multirow{3}{*}{ ATIR } & LTI & 1.19 & 0.0222 & 0.0115 & 1.86 & 1.48 & 1.33 & 0.517 & $10 \times 180$ \\
\hline & MTI & 1.24 & 0.0537 & 0.0230 & 4.33 & 3.33 & 2.93 & 0.428 & $11 \times 180$ \\
\hline & HTI & 1.17 & 0.160 & 0.178 & 13.7 & 14.4 & 14.7 & 1.11 & $10 \times 360$ \\
\hline \multirow[t]{2}{*}{ D12 } & MTI & 1.23 & 0.0534 & 0.0246 & 4.32 & 3.37 & 2.98 & 0.460 & $11 \times 180$ \\
\hline & HTI & 1.16 & 0.158 & 0.170 & 13.6 & 14.1 & 14.3 & 1.08 & $11 \times 360$ \\
\hline
\end{tabular}

Table 4

Turbulent flow characteristics for $U_{\infty}=1.2 \mathrm{~m} / \mathrm{s}$.

\begin{tabular}{llllllllll}
\hline Turbine & Case & $L[\mathrm{~m}]$ & $\varepsilon\left[\mathrm{m}^{2} \mathrm{~s}^{-3}\right]$ & $l[\mathrm{~m}]$ & $\eta[\mathrm{mm}]$ & $\lambda[\mathrm{mm}]$ & $R e_{\lambda}$ & $C_{0}$ & $\beta$ \\
\hline \multirow{2}{*}{ - } & LTI & 0.571 & $1.4110^{-5}$ & 0.581 & 0.570 & 22.2 & 393 & $5.5310^{-5}$ & 1.91 \\
& HTI & 0.539 & $1.1510^{-2}$ & 0.345 & 0.107 & 6.10 & 847 & $4.6210^{-3}$ & 1.72 \\
\hline IFREMER & HTI & 0.564 & $1.0010^{-2}$ & 0.372 & 0.110 & 6.41 & 871 & $4.2610^{-3}$ & 1.67 \\
\hline \multirow{3}{*}{ ATIR } & LTI & 0.629 & $1.7710^{-5}$ & 0.620 & 0.538 & 21.9 & 425 & $6.3910^{-5}$ & 1.80 \\
& MTI & 1.22 & $1.6010^{-4}$ & 0.967 & 0.310 & 17.6 & 826 & $2.9510^{-4}$ & 2.30 \\
& HTI & 0.553 & $1.1610^{-2}$ & 0.355 & 0.106 & 6.15 & 866 & $4.7110^{-3}$ & 1.70 \\
\hline \multirow{3}{*}{ D12 } & LTI & 0.495 & $1.2210^{-5}$ & 0.881 & 0.591 & 26.1 & 506 & $4.9610^{-5}$ & 1.91 \\
& MTI & 1.04 & $1.7610^{-4}$ & 0.862 & 0.303 & 16.6 & 778 & $3.1310^{-4}$ & 2.20 \\
& HTI & 0.588 & $9.9010^{-3}$ & 0.395 & 0.111 & 6.55 & 905 & $4.1310^{-3}$ & 1.68 \\
\hline
\end{tabular}

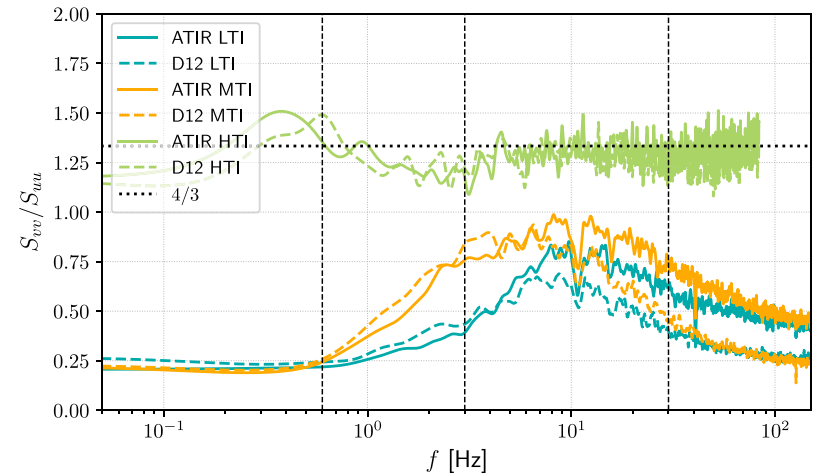

Fig. 11. Ratio of the transverse and streamwise velocity spectral densities, obtained at $U_{\infty}=1.2 \mathrm{~m} / \mathrm{s}$ for the three turbulence intensities.

is not surprising since the turbulent flow is not locally isotropic for the lower $I_{\infty}$ values. Indeed, the local isotropy assumption is valid if $S_{v v} / S_{u u}=4 / 3$ in the inertial subrange (Saddoughi and Veeravalli, 1994; Milne et al., 2013), which is not the case (see Fig. 11) for the LTI and MTI conditions. Nevertheless, the values obtained for the dissipation rates $\varepsilon$ are consistent with other studies (Blackmore et al., 2016; Medina et al., 2017).

From the dissipation rate $\varepsilon$, another characteristic length of the eddy size, the injection length scale $l$ (Blackmore et al., 2016; Medina et al., 2017), corresponding approximately to the scale at which energy enters the turbulent cascade from the mean flow, and two other scaling properties, Kolmogorov's dissipation scale $\eta$ and the Reynolds number $R e_{\lambda}$ based on Taylor length scale $\lambda$ (Pope, 2000), can be estimated:

$$
\begin{aligned}
l & \approx \frac{\sigma_{u_{\infty}}^{3}}{\varepsilon} \\
\eta & =\left(\frac{v}{\varepsilon}\right)^{1 / 4} \\
\lambda & =\sqrt{\frac{15 v}{\varepsilon}} \sigma_{u_{\infty}} \\
R e_{\lambda} & =\frac{\sigma_{u_{\infty}} \lambda}{v}
\end{aligned}
$$

where $v \approx 1.14110^{-6} \mathrm{~m}^{2} / \mathrm{s}$ is the water kinematic viscosity. As these quantities can be useful for comparison with other trials results, their values are also given in Table 4 as a matter of information. However, since Kolmogorov's law is not respected for the LTI and MTI cases, these estimations may not be accurate. At HTI, the values are similar for the four configurations.

\section{Performance evaluation}

The performances of the three scaled devices are evaluated using the power and thrust coefficients which are defined respectively by:

$C_{P}=\frac{Q \omega_{x}}{\frac{1}{2} \rho S \overline{u_{\infty}^{3}}}$

and :

$C_{T}=\frac{T}{\frac{1}{2} \rho \overline{u_{\infty}^{2}}}$

where $Q$ is the rotor torque, $T$ is the rotor thrust and $\rho$ is the fluid density. The upstream axial velocity $u_{\infty}$ here is the velocity measured by the LDV for each configuration. The overbar denotes the time average. As the blade profiles are confidential, all results were divided by a reference value, chosen for the LTI condition and a velocity $U_{\infty}$ :

- power coefficients and their standard deviations are divided by the maximum $C_{P}$ value;

- thrust coefficients and their standard deviations are divided by the $C_{T}$ value at the TSR of the maximum value of $C_{P}$.

The anonymised values will be identified by *. As the ATIR and D12 performances are compared to those of the IFREMER model in this section, the choice of the velocity case for the reference value was made in order to have the highest common Reynolds numbers (see Table 5). Thus, the selected velocities are $U_{\infty}=1.3 \mathrm{~m} / \mathrm{s}$ for the ATIR, $1.4 \mathrm{~m} / \mathrm{s}$ for the D12 and $1.2 \mathrm{~m} / \mathrm{s}$ for the IFREMER turbine, for which the Reynolds number $R e_{\infty}$ is between $4.20 \times 10^{5}$ and $4.39 \times 10^{5}$.

The results obtained for the time averaged power and thrust coefficients and their standard deviations, as a function of the TSR, are presented in the following subsections. 

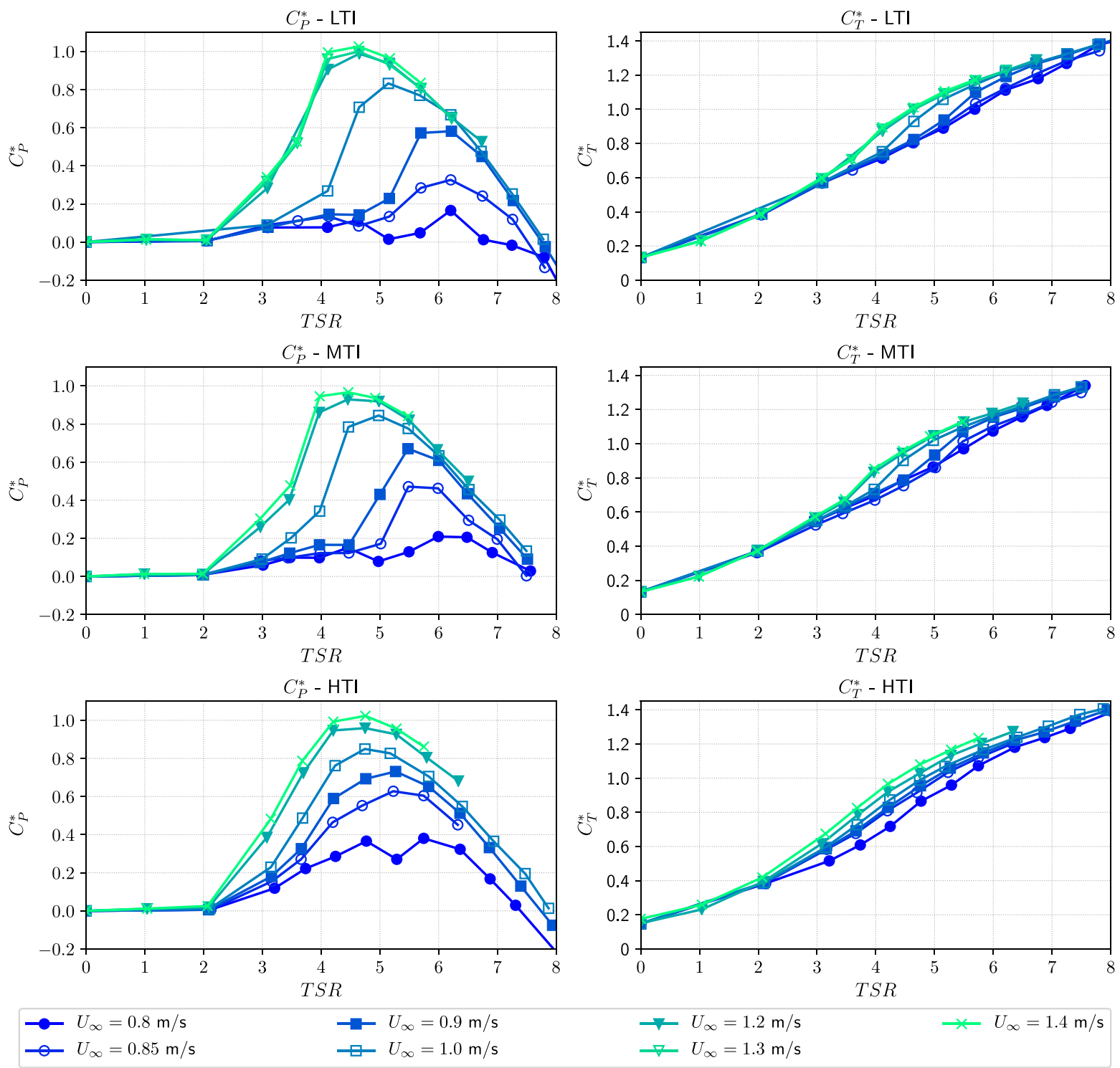

Fig. 12. Power and thrust coefficients for the three ambient turbulence intensity cases, obtained for the ATIR turbine.

Table 5

Upstream velocities and Reynolds numbers for the three scaled turbines.

\begin{tabular}{lll}
\hline Turbine & $U_{\infty}[\mathrm{m} / \mathrm{s}]$ & $R e_{\infty}$ \\
\hline \multirow{3}{*}{ ATIR } & 0.8 & $2.70 \times 10^{5}$ \\
& 1.0 & $3.38 \times 10^{5}$ \\
& 1.2 & $4.01 \times 10^{5}$ \\
& 1.3 & $\mathbf{4 . 3 9} \times \mathbf{1 0}$ \\
& 1.4 & $4.73 \times 10^{5}$ \\
\hline \multirow{2}{*}{ D12 } & 0.8 & $2.40 \times 10^{5}$ \\
& 1.0 & $3.00 \times 10^{5}$ \\
& 1.2 & $3.60 \times 10^{5}$ \\
IFREMER & 1.4 & $\mathbf{4 . 2 0} \times \mathbf{1 0}$ \\
& 0.8 & $2.90 \times 10^{5}$ \\
& 1.0 & $3.62 \times 10^{5}$ \\
\hline
\end{tabular}

The chosen reference cases are written in bold blue text

\subsection{Mean power and thrust coefficients}

\subsubsection{Magallanes Renovables' ATIR turbine}

Fig. 12 shows the power and thrust coefficients obtained for several upstream velocities and the three ambient turbulence intensities considered $\left(I_{\infty} \approx 1.5 \%, I_{\infty} \approx 3 \%\right.$ and $\left.I_{\infty} \approx 15 \%\right)$. For all turbulence intensities, the ATIR turbine operates properly for upstream velocities above $1.0 \mathrm{~m} / \mathrm{s}$. For these velocities the $C_{P}{ }^{*}$ curves tend to collapse into a single curve over the whole TSR range. For the lower upstream velocities $\left(U_{\infty} \leq 1.0 \mathrm{~m} / \mathrm{s}\right)$, an important Reynolds effect is observed. This Reynolds effect is mainly observable at the laboratory scale (see Table 2). For the smallest velocities $\left(U_{\infty} \leq 0.85 \mathrm{~m} / \mathrm{s}\right)$, the turbine hardly operates for the low and medium $I_{\infty}$. As expected, this Reynolds effect is largely attenuated for the highest turbulence intensity and the shapes of the performance curves are improved. For all $I_{\infty}$ configurations, the operating range is similar and falls within $4 \leq \mathrm{TSR} \leq 5.5$, but it has a slightly narrowed tendency for the LTI and MTI cases. For all turbulence intensity configurations and for velocities $\geq 1.2 \mathrm{~m} / \mathrm{s}$, the maximal $C_{P}{ }^{*}$ value is reached.

Very similar behaviours are observed for the thrust coefficients. Again a Reynolds effect is visible, although less important, for upstream velocities lower than $1.2 \mathrm{~m} / \mathrm{s}$ for the LTI and MTI. But beyond this velocity, the thrust coefficient curves are also superimposed. On the contrary, at HTI the curves do not superimpose. Fig. A.39 in Appendix shows the same curves (for TSR $\in[3 ; 8]$ ) with error bars denoting \pm the standard deviations. It can be seen that the differences between the $C_{T}{ }^{*}$ curves are larger than the standard deviations for the LTI 

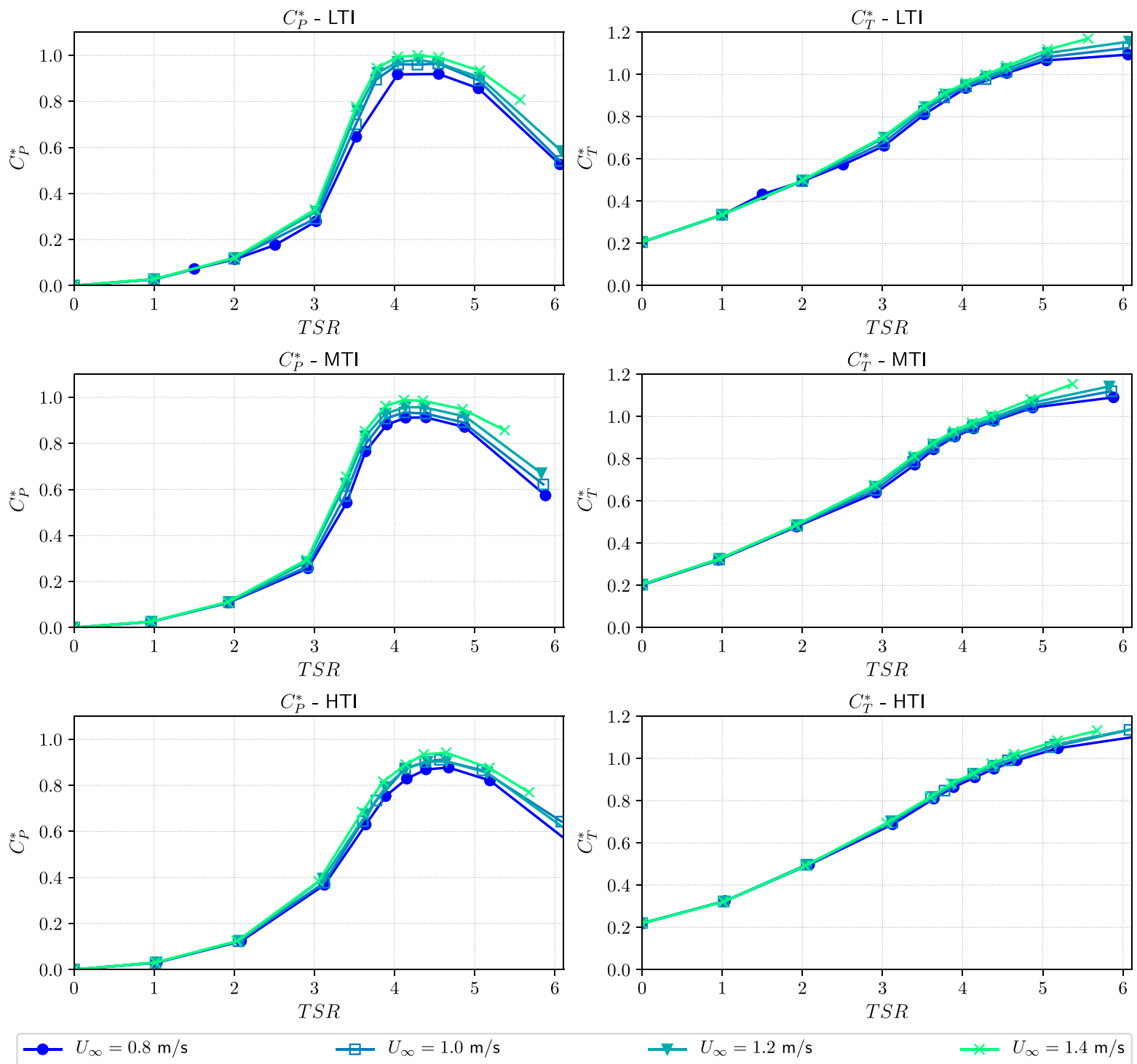

Fig. 13. Power and thrust coefficients for the three ambient turbulence intensity cases, obtained for the D12 turbine.

and MTI. From these plots, it is clear that the differences are not due to the standard deviations and uncertainties. For the HTI condition, the differences are smaller than the standard deviations, however the mean coefficients were estimated for a large dataset (with more than 40,000 samples). Thus, the differences are significant and due to a minor Reynolds effect. This was already presented by Gaurier et al. (2015). The large error bars for this HTI configuration (Fig. A.39) are fluctuating loads due to turbulence around a converged value. Besides, for the three turbulence intensities, the thrust coefficient keeps increasing with the TSR value. This observation differs slightly from the results of Mycek et al. (2014) but is very similar to the recent results of Gaurier et al. (2020).

\subsubsection{Sabella's D12 turbine}

Fig. 13 shows the power and thrust coefficients obtained for Sabella's D12 scaled turbine. The tests were carried out at the same turbulence intensities as for the ATIR and for only 4 velocities $\left(U_{\infty}=\right.$ $0.8,1.0,1.2$ and $1.4 \mathrm{~m} / \mathrm{s}$ ). For the three turbulence intensities, the device operates without any major Reynolds influence. Whatever the upstream velocity is, the $C_{P}{ }^{*}$ and $C_{T}{ }^{*}$ curves collapse (see Fig. 13). Both power and thrust coefficients have similar values at LTI, MTI and HTI, slightly lower for the later case. For a given ambient turbulence level, only a minor Reynolds effect is observable on the maximum values of power as explained by Gaurier et al. (2015). For the $C_{T}{ }^{*}$ curves at LTI, the standard deviations are at the order of magnitude of the differences, but the tendency is clear: a very small increase of the value for an increasing velocity, as depicted on the zoom of Fig. 13 presented in Fig. A.40 in Appendix. In that sense, the curves are collapsing towards the Reynolds independent values. For the $C_{P}{ }^{*}$ curves at LTI and for both the $C_{P}{ }^{*}$ and $C_{T}{ }^{*}$ curves at MTI and HTI (Fig. A.40), a similar behaviour seems to operate although the standard deviations are slightly larger to much larger than the differences. To our point of view, these curves are also collapsing towards the Reynolds independent values and the presented averaged data are converged. However, the standard deviations are slightly larger because the ambient turbulence imposes these load fluctuations. These two phenomena (collapsing towards the Reynolds independence and turbulence influence) appear on these results. Finally, for this turbine, the operating range is included in $3.75 \leq \mathrm{TSR} \leq 5.0$.

\subsubsection{Comparison}

The performances of both Magallanes Renovables and Sabella scaled turbines are now compared in Fig. 14 for the three turbulence intensities and several upstream velocities. For the ATIR, only the velocities above $1.2 \mathrm{~m} / \mathrm{s}$ were considered. These curves are compared with the 

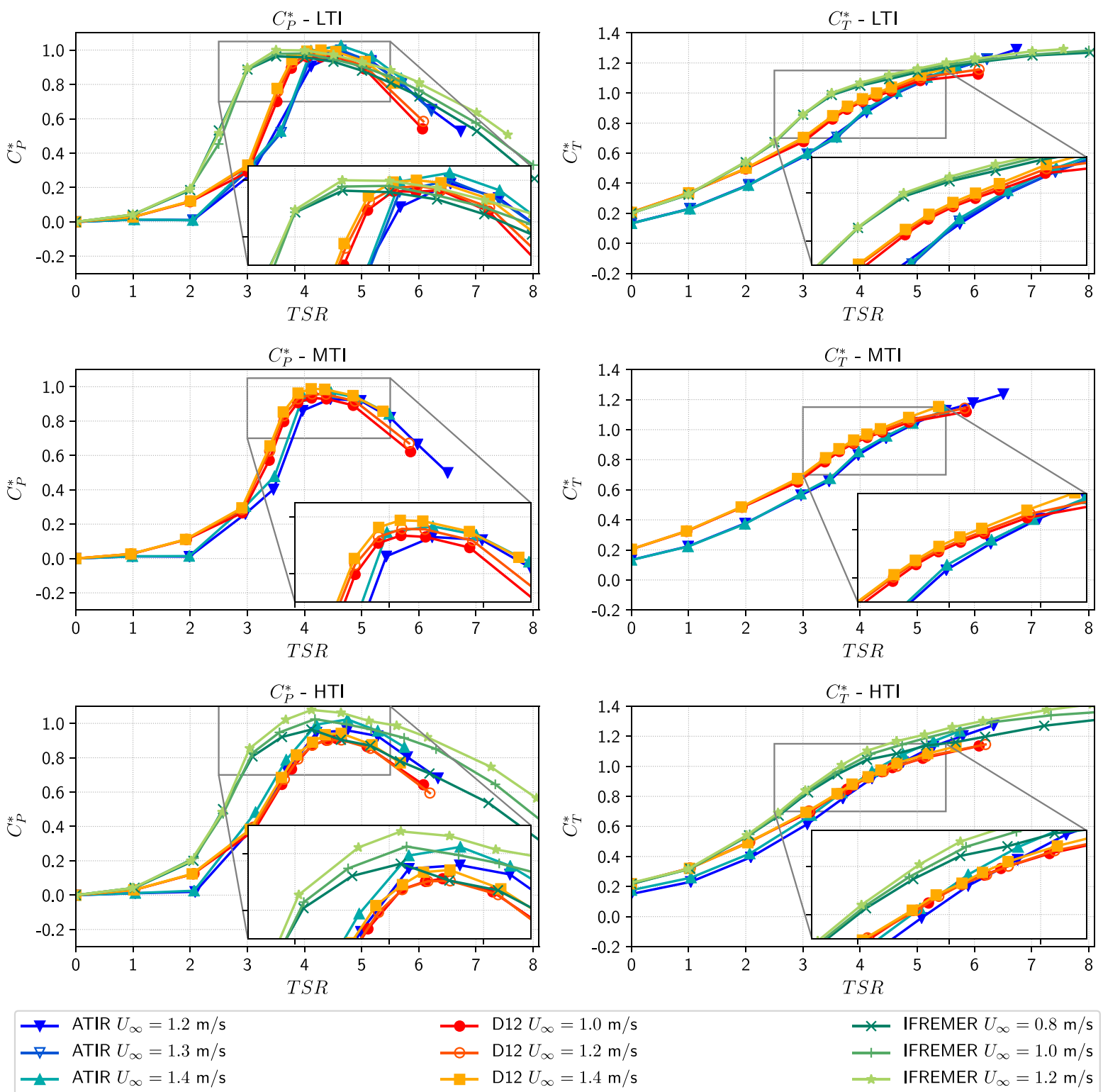

$*$ IFREMER $U_{\infty}=0.8 \mathrm{~m} / \mathrm{s}$

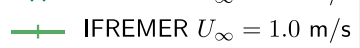

\#IFREMER $U_{\infty}=1.2 \mathrm{~m} / \mathrm{s}$

Fig. 14. Comparison of the power and thrust coefficients of the ATIR, D12 and IFREMER turbines for three ambient turbulence intensity cases.

existing results of the IFREMER turbine (Gaurier et al., 2019, 2020) (at LTI and HTI).

For the three turbulence intensities, both the ATIR and the D12 curves are close although the turbines have a very different design. It is however worth mentioning here that, this comparison is only valid for the anonymised results. The IFREMER turbine presents a larger operating range $(3.0 \lesssim \mathrm{TSR} \lesssim 5.0)$ than its two industrial counterparts. For the HTI, a Reynolds effect is more visible and the maximum $C_{P}{ }^{*}$ are slightly higher than for the LTI, contrary to the other scaled models. For the highest velocities, when the Reynolds effects are suppressed, the maximum of the $C_{P}{ }^{*}$ coefficient is reached around the same TSR values for all turbines: approximately 4.5 for the D12 and the ATIR and 4 for the IFREMER model. This result is not modified by the intensity of the ambient turbulence.

With the given anonymising procedure, the D12 thrust coefficients curves are slightly higher than the ATIR ones for TSR values below 5 and 4 at for the lower $I_{\infty}$ conditions and at HTI respectively. The results obtained for the IFREMER turbines are always a little higher than the two previous ones. For the three turbines however, the $C_{T}{ }^{*}$ curves have similar shapes. Some inflexions in the curves are observed for different
TSR values, this will need to be analysed in a near future. Numerical computations, such as those of Togneri et al. (2020) could be useful in that respect.

\subsection{Fluctuations}

\subsubsection{Magallanes Renovables' ATIR turbine}

Figs. 15 and 16 depict the standard deviations of the power and thrust coefficients, $\sigma_{C_{P}}{ }^{*}$ and $\sigma_{C_{T}}{ }^{*}$, and the normalised standard deviations, $\sigma_{C_{P}}{ }^{*} / C_{P}{ }^{*}$ and $\sigma_{C_{T}}{ }^{*} / C_{T}{ }^{*}$, for three upstream velocities and all the turbulence intensity configurations. The normalised $\sigma_{C_{P}}{ }^{*}$ was calculated for TSR $\geq 3$ because the power coefficients are close to zero for the first TSR values (see Fig. 12).

For the LTI and MTI, when the turbine is operating with a velocity $U_{\infty}=1.0 \mathrm{~m} / \mathrm{s}$, for which the Reynolds effect is still very important, $\sigma_{C_{P}}{ }^{*}$ and $\sigma_{C_{T}}{ }^{*}$ are larger for $3 \lesssim \mathrm{TSR} \lesssim 5$ than the corresponding values for the higher upstream velocities. This strongly suggests that important "dynamic stall" or similar unsteady hydrodynamics features are present at some location on the blade due to this Reynolds effect.

For the higher incoming velocities (i.e. $U_{\infty} \geq 1.2 \mathrm{~m} / \mathrm{s}$ ), the turbulence intensity has an important influence on ${\sigma_{C}}_{C_{P}}{ }^{*}$ and $\sigma_{C_{T}}{ }^{*}$. Indeed, for 


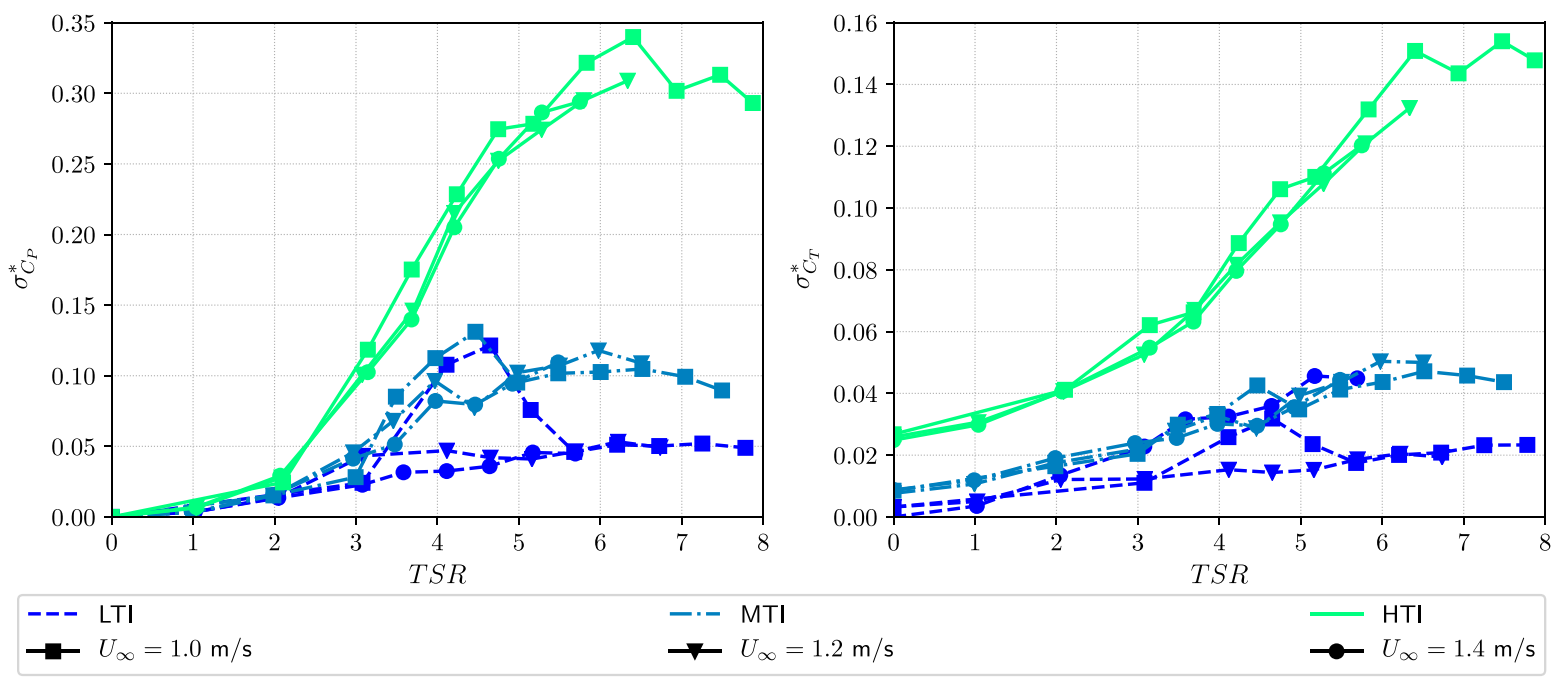

Fig. 15. Standard deviations of the power (left) and thrust (right) coefficients for the three ambient turbulence intensity cases, obtained for the ATIR turbine.

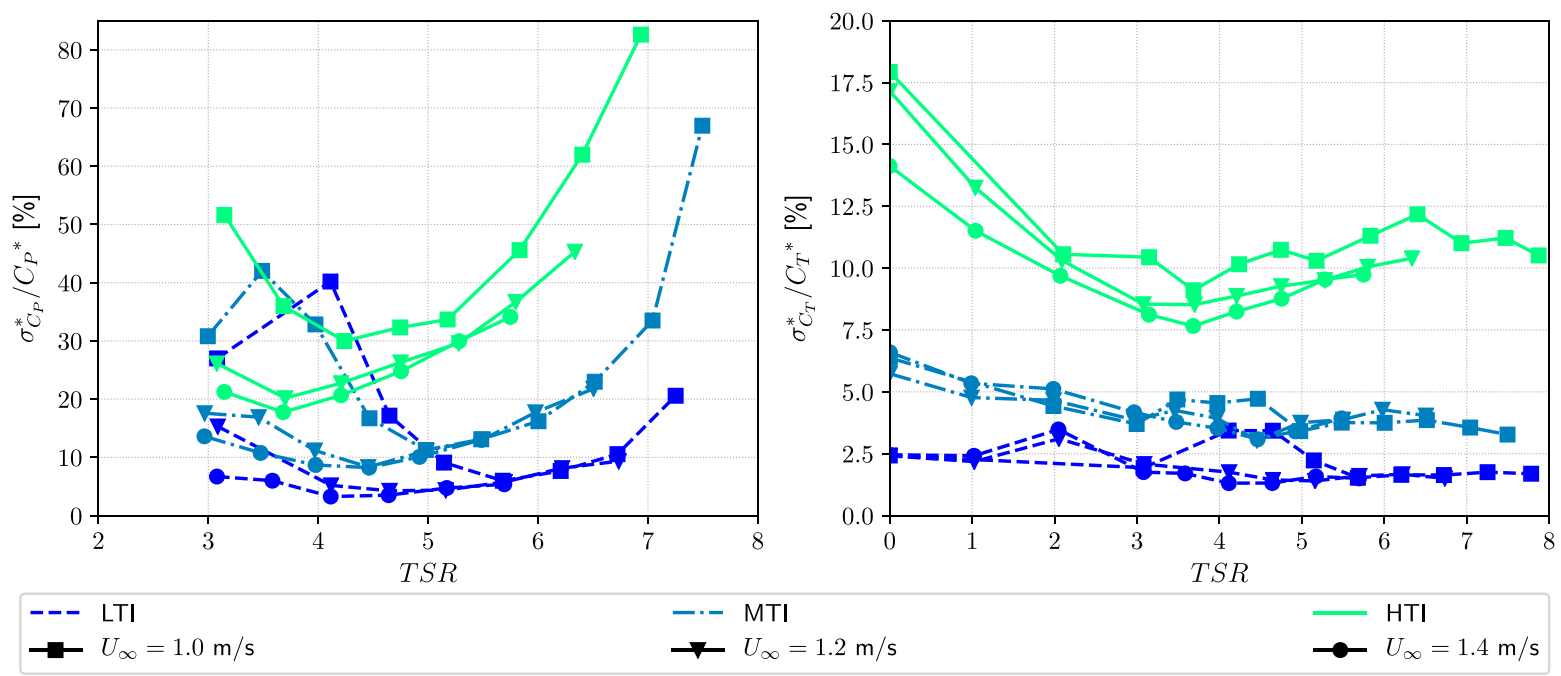

Fig. 16. Normalised standard deviations of the power (left) and thrust (right) coefficients for the three ambient turbulence intensity cases, obtained for the ATIR turbine.

those velocities and in the operating range of TSR ( $4 \leq$ TSR $\leq 5.5$ ), the $\sigma_{C_{P}}{ }^{*}$ increase from $\approx 4-5 \%$ at LTI, to $\approx 9-13 \%$ at MTI and to $\approx 20-$ $-30 \%$ at HTI. This corresponds to approximately a multiplication by 6 between the lowest and highest turbulence intensities. The standard deviations of the thrust coefficient are about half the $\sigma_{C_{P}}{ }^{*}$ values but the same multiplication factor can be observed, with values going from $\approx 1.5 \%$ at LTI to $\approx 8-9 \%$ at HTI.

The normalised standard deviations of the rotational speed, for the same configurations, are presented in Fig. 17. These values are small, with a maximum of $0.5 \%$. Similar evolutions can be observed for the LTI and MTI. As for $\sigma_{C_{P}}{ }^{*}$ and $\sigma_{C_{T}}{ }^{*}$, a peak can be observed in the range $3 \lesssim \mathrm{TSR} \lesssim 5$ for $U_{\infty}=1.0 \mathrm{~m} / \mathrm{s}$. At HTI, for $U_{\infty} \geq 1.2 \mathrm{~m} / \mathrm{s}$, the standard deviations are approximately three times higher in the operating TSR range.

\subsubsection{Sabella's D12 turbine}

As for the ATIR scaled turbine, the ambient turbulence intensity has an important influence on the standard deviations of $C_{P}{ }^{*}$ and $C_{T}{ }^{*}$ (see Figs. 18 and 19). For all velocities and in the operating range of TSR $(3.75 \leq$ TSR $\leq 5)$, the normalised $\sigma_{C_{P}}{ }^{*}$ increase from $\approx 3-6 \%$ at LTI to $\approx 7-12 \%$ at MTI to $\approx 15-30 \%$ at HTI. Thus, $\sigma_{C_{P}}{ }^{*}$ is five times larger for the highest turbulence intensity than for the LTI. A multiplication by approximately 6 to 9 is observed for the $\sigma_{C_{T}}{ }^{*}$; with values going from

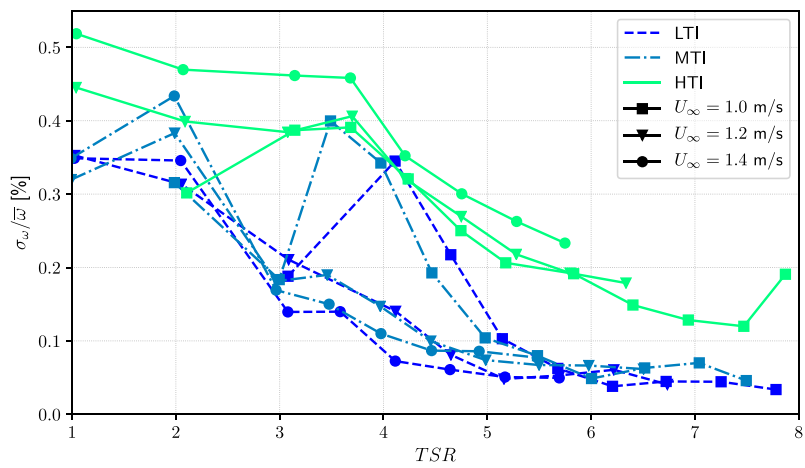

Fig. 17. Standard deviations of the rotational speed for the three ambient turbulence intensity cases, obtained for the ATIR turbine.

$\approx 1.5 \%$ at LTI to $\approx 10-13 \%$ at HTI. In addition, the normalised $\sigma_{C_{P}}{ }^{*}$ and $\sigma_{C_{T}}{ }^{*}$ curves superimpose for the three turbulence intensity cases and for all velocities.

The normalised standard deviations of the rotational speed are also low for this turbine (see Fig. 20), with a maximum of $0.3 \%$ at HTI. For 


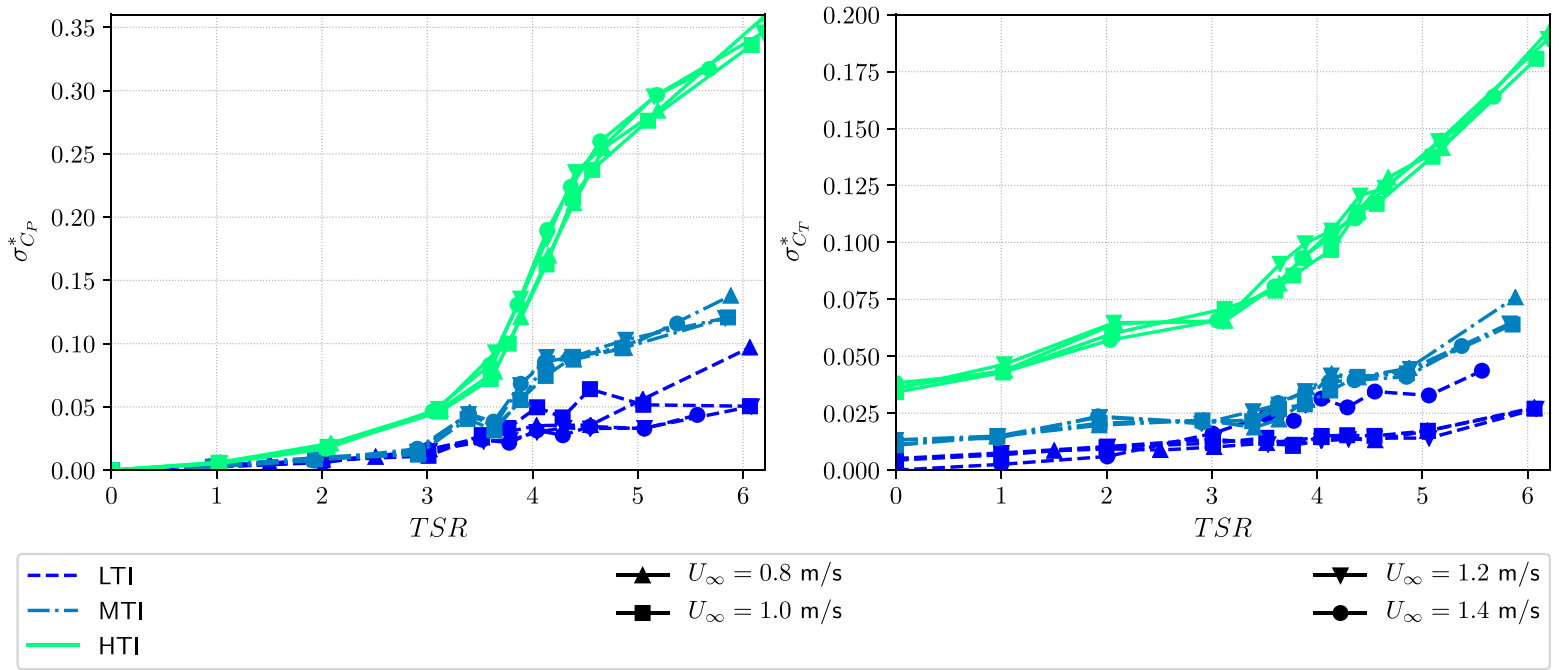

Fig. 18. Standard deviations of the power (left) and thrust (right) coefficients for the three ambient turbulence intensity cases, obtained for the D12 turbine.
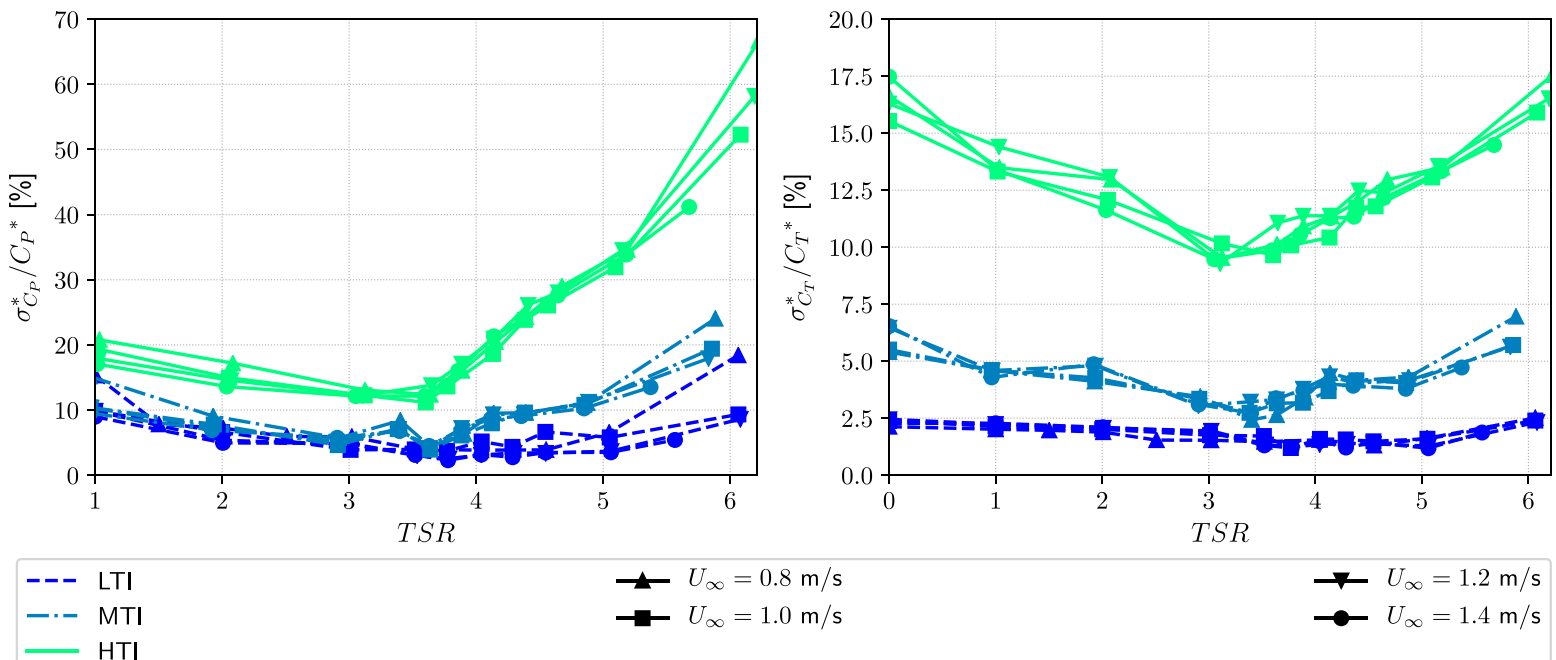

Fig. 19. Normalised standard deviations of the power (left) and thrust (right) coefficients for the three ambient turbulence intensity cases, obtained for the D12 turbine.

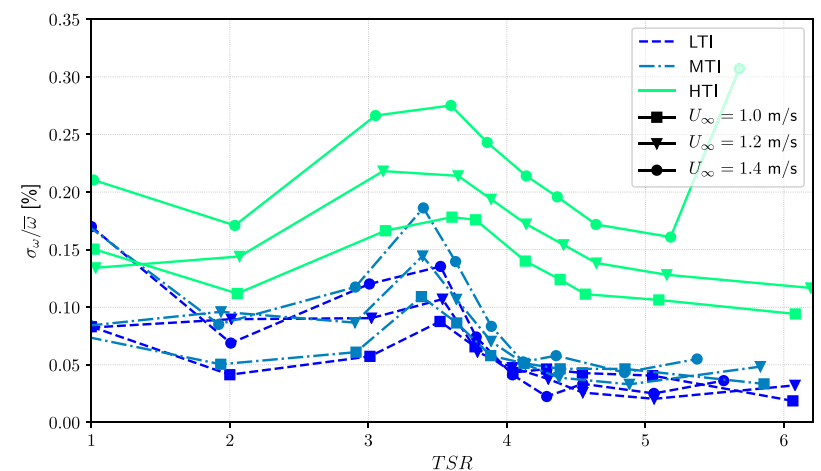

Fig. 20. Standard deviations of the rotational speed for the three ambient turbulence intensity cases, obtained for the D12 turbine.

all $I_{\infty}$ configurations, the maximum is reached for TSR $\approx 3.5$, which corresponds to the TSR where the normalised $\sigma_{C_{P}}{ }^{*}$ are the lowest. The standard deviations curves are similar for the low and medium $I_{\infty}$ and all velocities but they are 2 to 5 times higher at HTI, in the operating TSR range.

\subsubsection{Comparison}

A comparison of the standard deviations for the ATIR, D12 and IFREMER turbines is presented in Figs. 21 and 22. It is very interesting to observe that the $\sigma_{C_{P}}{ }^{*}$ and $\sigma_{C_{T}}{ }^{*}$ curves have similar shape and values for the three turbines.

For the $\sigma_{C_{P}}{ }^{*}$ at LTI, a dynamic load seems to appear at TSR $=2.5$ for all velocities for the IFREMER turbine. This precise aspect will need to be studied in deeper detail. For the MTI, the ATIR curves are close to the D12 ones for TSR $\geq 4$. At HTI, the D12 standard deviations grow faster than the other two turbines for TSR $\geq 3.5$. The ATIR and IFREMER turbines have very similar curves. For the optimal TSR value of each turbine, which lies approximately at $4.0 \lesssim \mathrm{TSR} \lesssim 4.5$, all the three turbines have a normalised $\sigma_{C_{P}}{ }^{*}$ of $\approx 25 \%$.

Regarding the standard deviation of the thrust coefficient, the $\sigma_{C_{T}}{ }^{*}$ at LTI are all concentrated in a narrow range between $1 \%$ to $2 \%$ for TSR $\in[3,5]$. For the MTI case, the ATIR curves are close to the D12 ones for TSR $\leq 5$. For the highest ambient turbulence configuration, the $\sigma_{C_{T}}{ }^{*}$ of the three turbines evolves similarly for TSR $\leq 3$. For larger TSR values, particularly in the operating range, the ATIR's results are lower than those of the IFREMER and D12 turbines.

To conclude, the ambient turbulence clearly has a dominant role on the turbine load fluctuations as already shown by Mycek et al. (2014), Blackmore et al. (2016) and other authors. At first glance, the turbine 

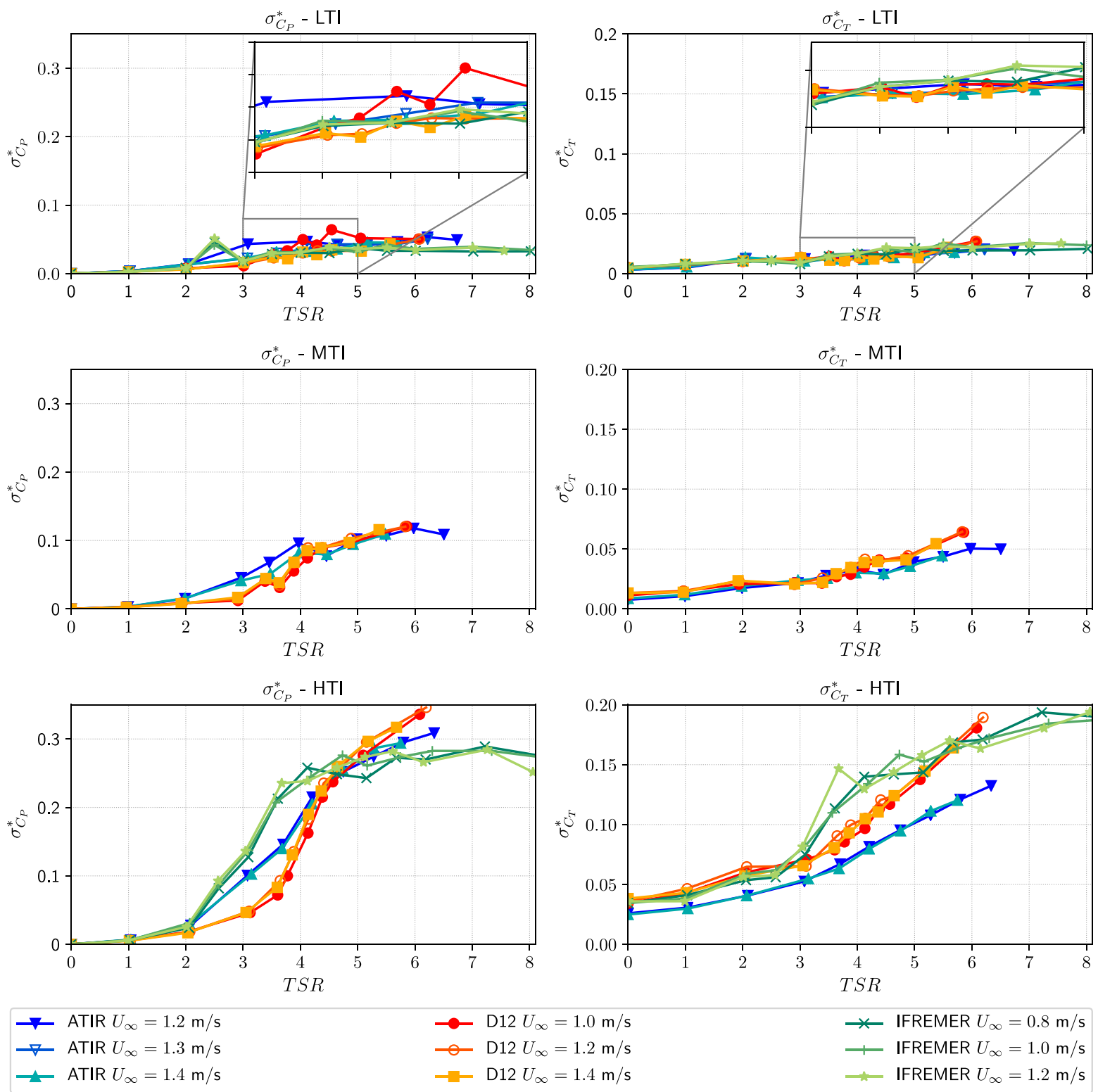

Fig. 21. Comparison of the standard deviations of the power and thrust coefficients of the ATIR, D12 and IFREMER turbines for three ambient turbulence intensity cases.

solidity, blade profile and blade number do not seem to have a major influence on the load fluctuations. Although the present results are clearly consistent with this hypothesis, this last conclusion needs deeper studies to be confirmed. One alternative would be that the drive train of the IFREMER nacelle (used for all the three scaled turbines) might have a larger influence on the obtained results, hiding the differences due to the rotor influence. Such conclusions would have a major influence on the fatigue of the blades and the other components of the turbines. This can impact significantly the turbines design and hence their cost (Pinon et al., 2017).

\section{Spectral analysis}

\subsection{Power spectral density and coherence}

In addition to the impact of the ambient turbulence on the power and thrust coefficient, the spectral contents of the rotor and blade root loads were studied. For this purpose, their power spectral densities (PSD) were computed (using the welch function of Python, as in Section 3) for the three turbulence intensities and several TSR values.
Since the industrial turbines performances are confidential, the blade root loads (axial forces $F_{x i}$ and edgewise bending moments $M_{x i}$ ), the torque and the thrust are also divided by a reference value, chosen for the same case as in Section 4:

$$
\begin{aligned}
Q^{*} & =\frac{Q}{Q_{\mathrm{ref}}} ; \quad M_{x i}^{*}=\frac{M_{x i}}{\sum_{i=1}^{N_{b}} M_{x i} \mathrm{ref}} ; \\
T^{*} & =\frac{T}{T_{\mathrm{ref}}} ; \quad F_{x i}^{*}=\frac{F_{x i}}{\sum_{i=1}^{N_{b}} F_{x i} \mathrm{ref}} ;
\end{aligned}
$$

where $i$ is the blade index and $N_{b}$ is the number of blades. The subscripts $r$ ef denotes the reference values.

In order to further analyse the frequency dependency, the magnitude squared coherences (MSC) between the rotor loads and the upstream flow velocity or the rotational speed were also estimated. For two signals $X(t)$ and $Y(t)$, the magnitude squared coherence $\gamma_{X Y}^{2}$ is defined as (Stoica and Moses, 2005):

$\gamma_{X Y}^{2}=\frac{\left|S_{X Y}(f)\right|^{2}}{S_{X X}(f) S_{Y Y}(f)}$ 

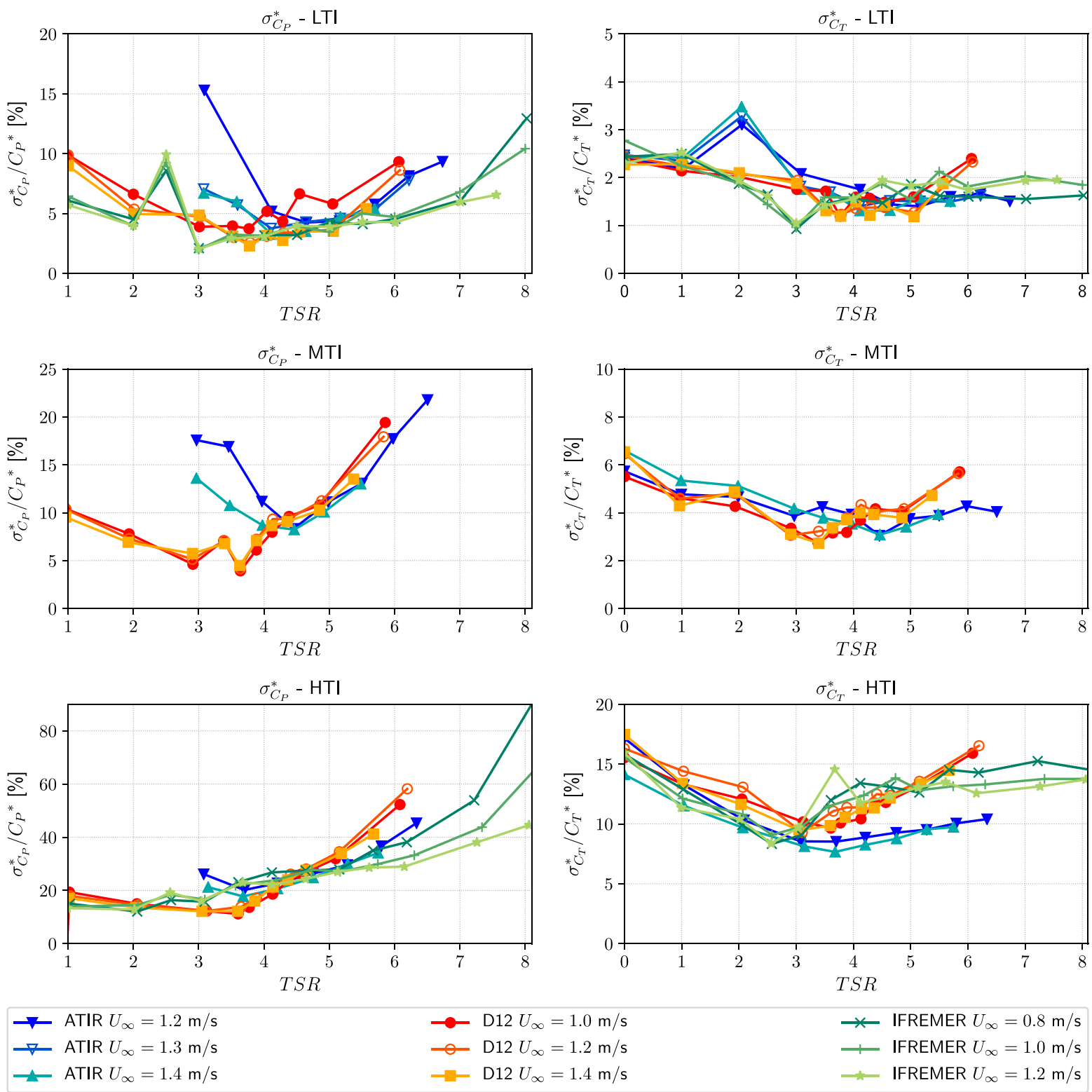

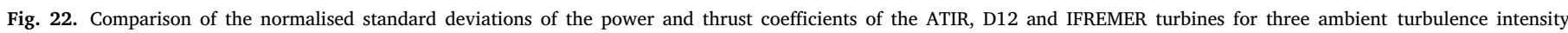
cases.

where $S_{X X}$ and $S_{Y Y}$ are the power spectral densities of $X$ and $Y$ respectively, $S_{X Y}$ is the cross spectral density of $X$ and $Y$. The MSC function indicates the extent to which the signal $Y(t)$ is related to $X(t)$. This function varies between 0 and 1 . A MSC equal to one indicates that $X(t)$ and $Y(t)$ are linearly related. If $\gamma_{X Y}^{2}=0$, the signals are unrelated. In this study the MSC were estimated with the scipy.signal.coherence function of Python.

To compute the MSC $\gamma_{Q u}^{2}$ between the torque $Q$ and the upstream flow velocity $u_{\infty}$, the velocity measurements were interpolated on the same time samples as the load ones (with a sampling frequency of $120 \mathrm{~Hz}$ ). The same method was applied to compute the MSC $\gamma_{T u}^{2}$ between the thrust $T$ and $u_{\infty}$. Furthermore, since $u_{\infty}$ is measured at $1.4 \mathrm{~m}$ upstream of the turbine, there is a time lag between the flow speed and the turbine measurements. Similarly to Medina et al. (2017), this time lag was estimated using the cross correlation between $T$ and $u_{\infty}$ and was taken into account for the computation of the MSC.

The results obtained for the ATIR and D12 turbines, with an upstream velocity of $1.2 \mathrm{~m} / \mathrm{s}$, are presented in the following subsections.

\subsection{Magallanes Renovables' ATIR turbine}

The torque and thrust PSD at HTI are presented in Figs. 23 and 24 for three TSR values: at the nominal operating point (TSR $\approx 4.5$ ), close to the run away point (TSR $\approx 6$ ) and for a TSR with dynamic stall effects (TSR $\approx 3$ ). The frequency is normalised by the turbine mean rotational frequency $f_{0}$. For TSR $\approx 4.5$ and 6 , the torque spectral densities are relatively close (Fig. 23). Two main peaks can be observed: at $f_{0}$ and $3 f_{0}$. The latter corresponds to the passage of the three blades in front of the mast (Fernandez-Rodriguez et al., 2014; Payne et al., 2018; Martinez et al., 2018). At $6 f_{0}$ a harmonic of the $3 f_{0}$ peak is identifiable. For TSR $\approx 6$ a peak is also visible at $2 f_{0}$. For TSR $\approx 3, S_{Q Q}$ is higher for $f / f_{0}>0.2$ and the $f_{0}, 3 f_{0}$ and $6 f_{0}$ peaks are less pronounced. The peak at $3 f_{0}$ is also larger. For the three TSR values, harmonics of the $f_{0}$ peak can be observed at high frequencies. The evolution of $S_{T T}$ (Fig. 24) is relatively similar but harmonics of the $3 f_{0}$ peak are present at $9 f_{0}, 12 f_{0}$ and $15 f_{0}$ for TSR $\approx 6$. At TSR $\approx 4.5$, there are only two main peaks, at $3 f_{0}$ and $6 f_{0}$, and a small one at $9 f_{0}$. Lastly, at TSR $\approx 3$, a large peak around $3 f_{0}$ and harmonics of $f_{0}$ are identifiable. 


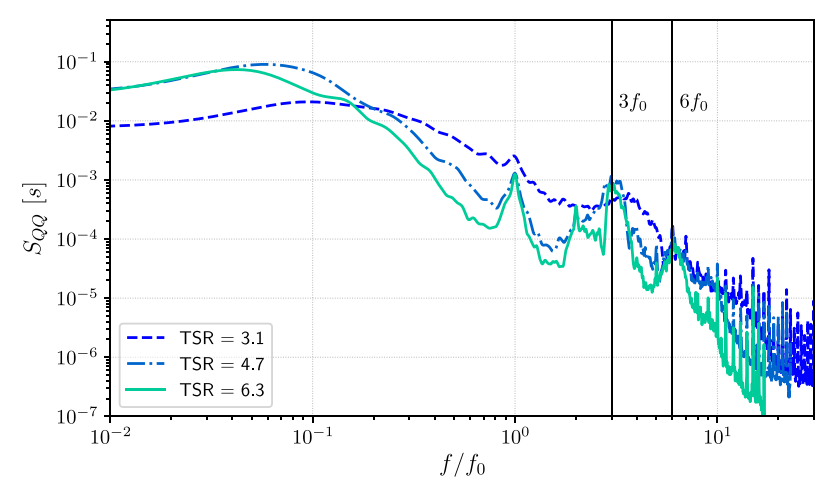

Fig. 23. Torque power spectral densities obtained for the ATIR turbine for three TSR, $U_{\infty}=1.2 \mathrm{~m} / \mathrm{s}$ and the HTI case.

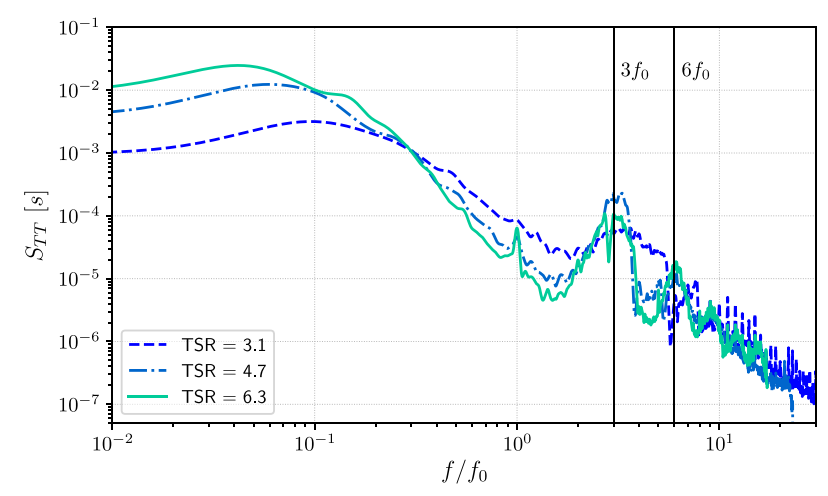

Fig. 24. Thrust power spectral densities obtained for the ATIR turbine for three TSR, $U_{\infty}=1.2 \mathrm{~m} / \mathrm{s}$ and the HTI case.

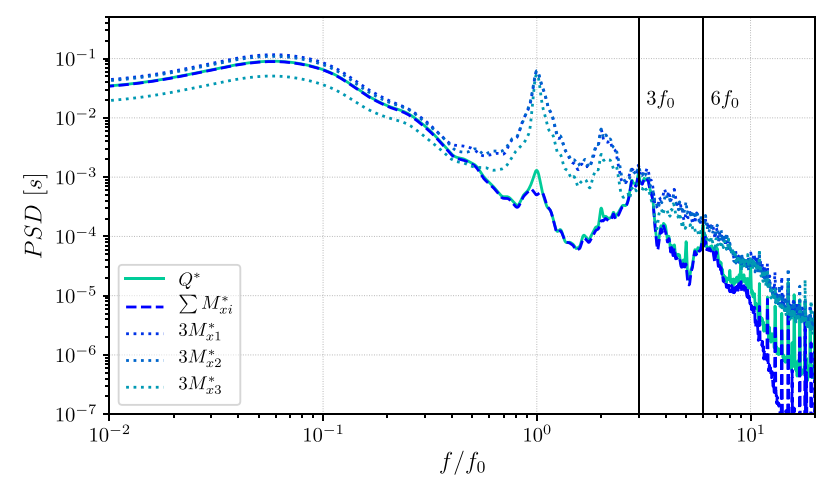

Fig. 25. Comparison of the power spectral densities of the rotor torque and the edgewise blade root bending moments obtained for the ATIR turbine for TSR $=4.7$, $U_{\infty}=1.2 \mathrm{~m} / \mathrm{s}$ and the HTI case.

Fig. 25 shows the PSD of the torque, the blade root edgewise bending moments for each blade (multiplied by 3 ) and their sum. The spectral densities of the blade bending moments are similar but some discrepancies can be observed for the third blade. Three peaks are identifiable: at $f_{0}$ (due to the blade passage) and the harmonics $2 f_{0}$ and $3 f_{0}$. For $f<0.5 f_{0}$, the PSD of $\sum M_{x i}^{*}$ has the same evolution as each $M_{x i}^{*}$. At higher frequencies, the amplitude of the overall moment spectrum is smaller except at $3 f_{0}$. This is due to the phase differences of the blade moments: their contributions to the total bending moment offset each other, particularly at $f_{0}$. There is a good agreement between the torque and the sum of the bending moments PSD for frequencies below $10 f_{0}$. Similar observations can be made about the thrust and the axial blade root forces PSD (see Fig. 26). However, the three blade force

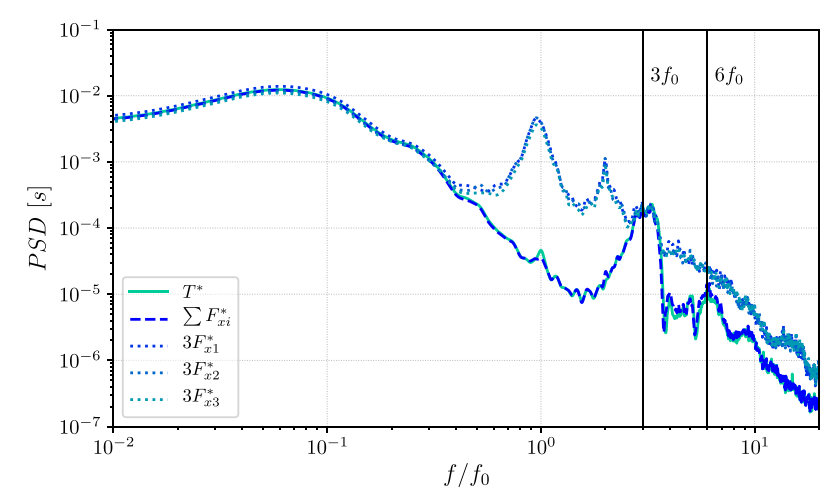

Fig. 26. Comparison of the power spectral densities of the rotor thrust and the axial blade root forces obtained for the ATIR turbine for TSR $=4.7, U_{\infty}=1.2 \mathrm{~m} / \mathrm{s}$ and the HTI case.

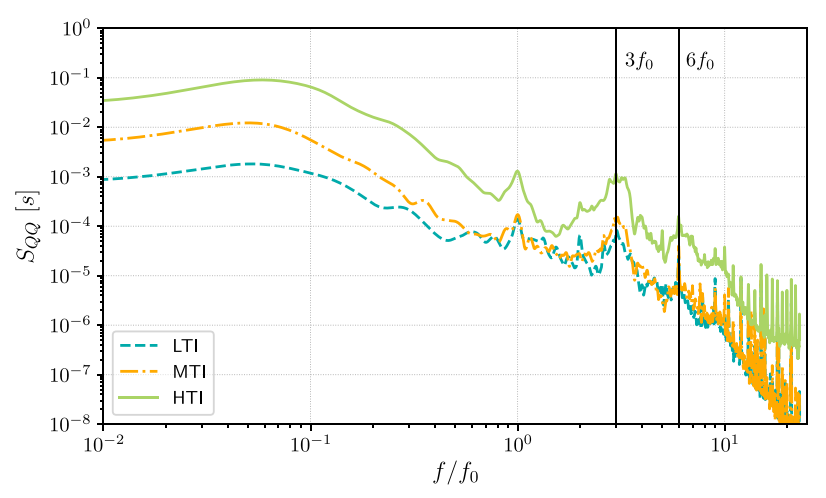

Fig. 27. Torque power spectral densities obtained for the ATIR turbine for the three turbulence intensity cases at $U_{\infty}=1.2 \mathrm{~m} / \mathrm{s}$ and TSR $\approx 4.5$.

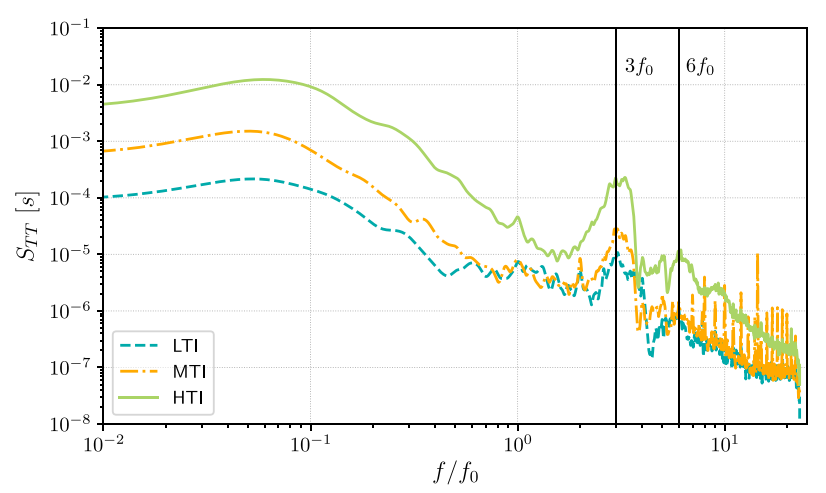

Fig. 28. Thrust power spectral densities obtained for the ATIR turbine for the three turbulence intensity cases at $U_{\infty}=1.2 \mathrm{~m} / \mathrm{s}$ and TSR $\approx 4.5$.

curves are almost identical and their sum is in good agreement with the thrust spectrum for the whole frequency range.

In order to study the influence of the ambient turbulence on the spectral content of the rotor loads, the torque and thrust power spectral densities were computed at the nominal TSR operating point for the three $I_{\infty}$ cases. These results are presented in Figs. 27 and 28.

As the velocity PSD shown in Fig. 9, the amplitudes of $S_{Q Q}$ and $S_{T T}$ increase with the turbulence intensity at low frequencies. This result is similar to what Payne et al. (2018) obtained for the spectral densities of the power and thrust coefficients. At LTI and MTI, the amplitudes are close for $f / f_{0} \geq 0.7$. For the torque PSD, three main peaks are visible for all the turbulence intensity cases: at $f_{0}, 3 f_{0}$ and $6 f_{0}$. For the thrust PSD, the main peak is located at $3 f_{0}$. Its harmonics at $6 f_{0}$ and $9 f_{0}$ (only 

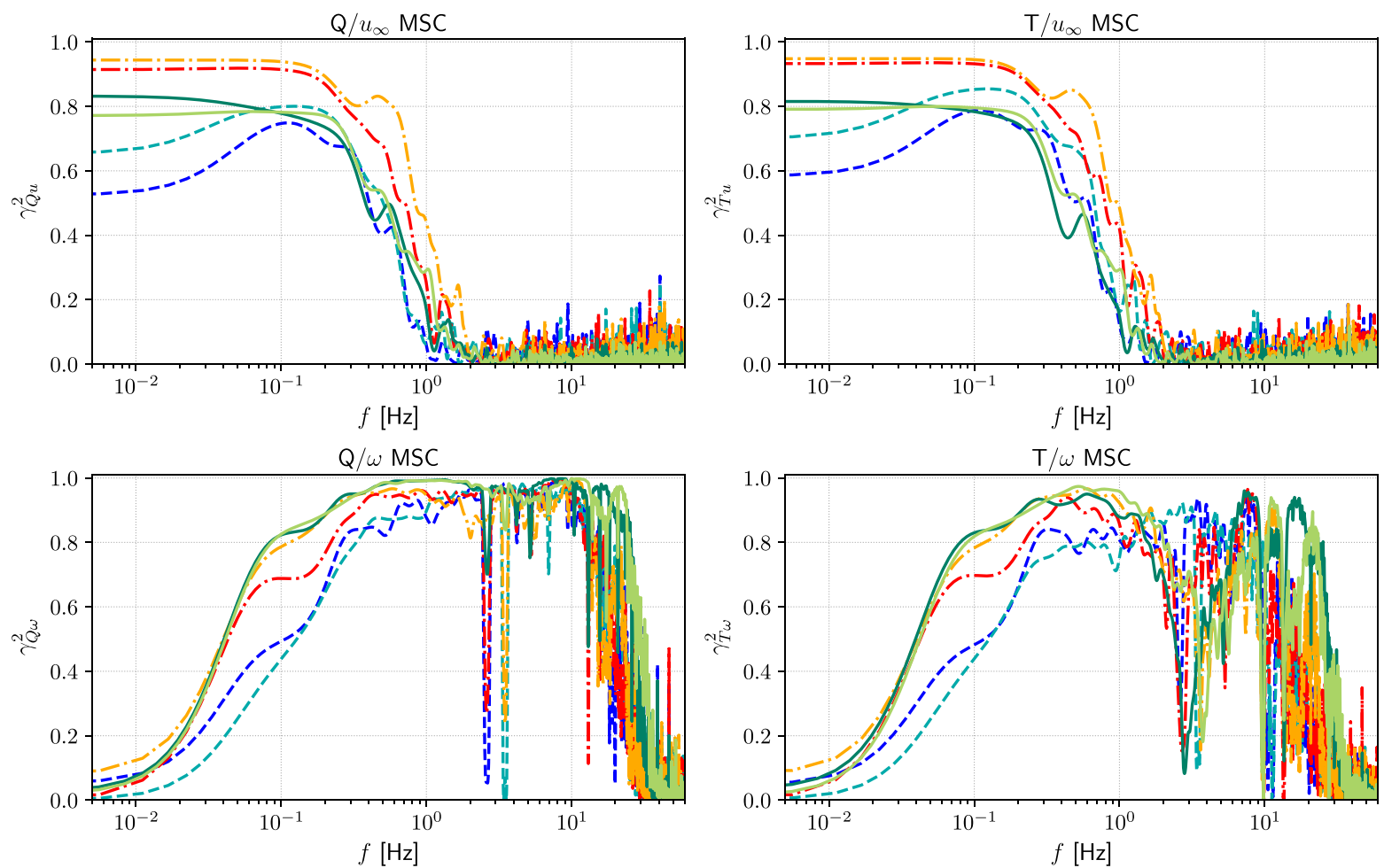

$$
\begin{array}{ll}
--- & \mathrm{LTI}-\mathrm{TSR}=4.5 \\
--- & \mathrm{LTI}-\mathrm{TSR}=6
\end{array}
$$$$
\begin{aligned}
& -\cdot-\mathrm{MTI}-\mathrm{TSR}=4.5 \\
& -\cdot-\mathrm{MTI}-\mathrm{TSR}=6
\end{aligned}
$$

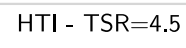

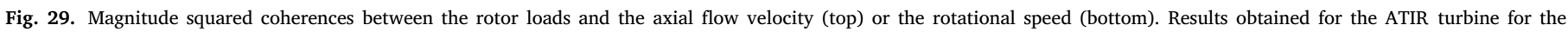
three turbulence intensity cases at $U_{\infty}=1.2 \mathrm{~m} / \mathrm{s}$ and two TSR values (TSR $\approx 4.5$ and TSR $\approx 6$ ).

for the HTI case) are also visible. For the MTI condition, harmonics of $f_{0}$ are identifiable at high frequencies.

The two top plots of Fig. 29 show the magnitude squared coherences between the flow velocity $u_{\infty}$ and the torque $\gamma_{O u}^{2}$ (left) and the thrust $\gamma_{T u}^{2}$ (right), for two TSR values and the three turbulence intensity configurations. It appears that the evolutions of $\gamma_{O u}^{2}$ and $\gamma_{T u}^{2}$ are almost identical and that, except for the LTI case at low frequencies, the TSR has little effect on the coherences. The latter result is consistent with those of Gaurier et al. (2019). As observed by Medina et al. (2017), three regions can be identified: at low frequencies $(f<0.2 \mathrm{~Hz})$ the coherences are relatively high and reach their maximum; for $f \in[0.2,2] \mathrm{Hz}$ the MSC curves drop and then remain near zero at higher frequencies. At low frequencies, the coherence maxima are between 0.75 and 0.95 , thus the torque and thrust variations are related to the velocity variations. This results is in agreement with the observations of Fernandez-Rodriguez et al. (2014), Medina et al. (2017) and Payne et al. (2018). For frequencies higher than $\approx 1-2 \mathrm{~Hz}$ the rotor load variations are no longer related to the flow velocity. It seems that the load and velocity variations are correlated only for time scales larger than $\approx 0.5-1 \mathrm{~s}$, which corresponds to length scales larger than $\approx 0.6-1.2 \mathrm{~m}$ (using Taylor's hypothesis). As the scaled turbine diameter is $0.676 \mathrm{~m}$, the rotor loads variations seem only related to the eddies larger than the turbine. Thus the turbine may be acting as a low-pass filter as hypothesised by Gaurier et al. (2019). Furthermore, at low frequencies, the coherences are higher for the MTI case. This may be due to the fact that the flow characteristic length scale $L$ is higher for this turbulence intensity whereas its value is approximately the same for the LTI and HTI conditions (see Table 4).

The two bottom plots of Fig. 29 present the MSC between the rotational velocity $\omega$ and the torque $\gamma_{Q \omega}^{2}$ (left) and the thrust $\gamma_{T \omega}^{2}$ (right), for two TSR values and the three turbulence intensity configurations. Three zones can also be observed for $\gamma_{Q \omega}^{2}$ : for frequencies below $1 \mathrm{~Hz}$ the coherences increase; for $f \in[1,10] \mathrm{Hz}$ the MSC curves remain close to 1 (except for a drop at $f=f_{0}$ ); for $f>10 \mathrm{~Hz}$ the coherences decrease. The evolution of $\gamma_{T \omega}^{2}$ is similar but the drop for $f \approx f_{0}$ happen for a larger frequency range. For both MSC functions the coherence increase at low frequencies with the turbulence intensity. These results confirm that, at low frequencies, the torque and thrust variations are mainly related to the flow velocity. For $f>1 \mathrm{~Hz}$, they are significantly more closely correlated to the rotational speed control unit of the scaled turbine.

\subsection{Sabella's D12 turbine}

Similarly to the results presented for the ATIR turbine, Figs. 30 and 31 show the torque and thrust PSD obtained for the D12 model, for three TSR values and the HTI case.

The torque spectral densities for TSR $\approx 4.5$ and 6 evolve similarly (Fig. 30). The largest peak is located at $5 f_{0}$ and corresponds to the passage of the five blades of the scaled turbine. A harmonic of this peak is identifiable at $10 f_{0}$, particularly for TSR $\approx 4.5$. A peak at the rotational frequency and its harmonics can also be found. The peak visible at $\approx 2.6 f_{0}$ is believed to be caused by the blade supports used for this scaled model (see Fig. 8). For TSR $\approx 3, S_{Q Q}$ is higher for $f / f_{0} \geq 1$. The $f_{0}$ and $5 f_{0}$ peaks are also visible but they are less pronounced.

The evolution of $S_{T T}$ (Fig. 31) is relatively similar but the peaks at $f_{0}$ and $2 f_{0}$ are visible only at TSR $\approx 6$. For the latter TSR value, there is also a harmonic of the $5 f_{0}$ peak present at $15 f_{0}$. For TSR $\approx 3$, there is no peak related to the rotational frequency and a drop instead of a peak is visible at $5 f_{0}$.

Fig. 32 shows a comparison of the PSD of the torque, the blade root edgewise bending moment for one blade (multiplied by 5) and the sum of the edgewise moments measured by the three load cells (see Fig. 8). For the PSD of $M_{x 1}^{*}$, several peaks are identifiable: at $f_{0}$ (due to the blade passage) and its harmonics, particularly at $2 f_{0}, 3 f_{0}$ and $4 f_{0}$. The peak at $\approx 2.6 f_{0}$ is not visible. There is a good agreement between the torque and the sum of the bending moments PSD for frequencies 


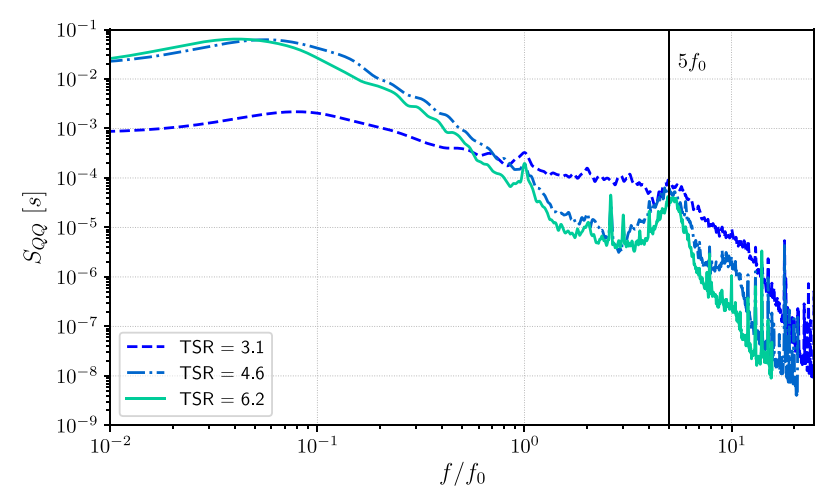

Fig. 30. Torque power spectral densities obtained for the D12 turbine for three TSR, $U_{\infty}=1.2 \mathrm{~m} / \mathrm{s}$ and the HTI case.

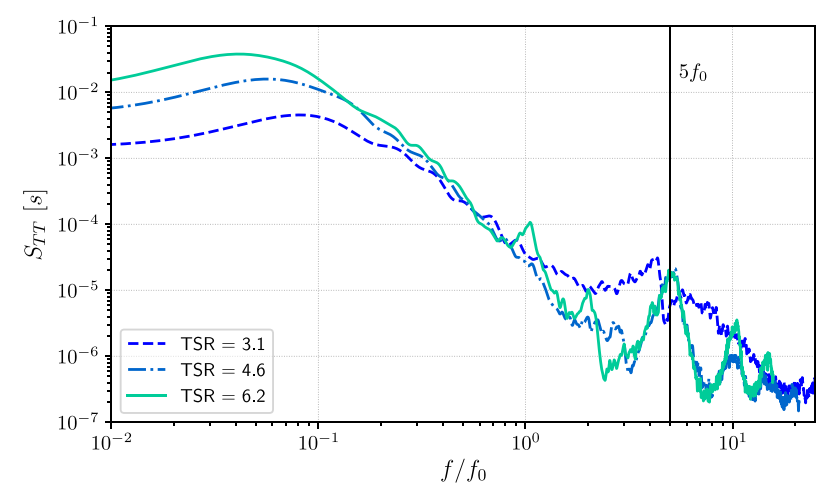

Fig. 31. Thrust power spectral densities obtained for the D12 turbine for three TSR, $U_{\infty}=1.2 \mathrm{~m} / \mathrm{s}$ and the HTI case.

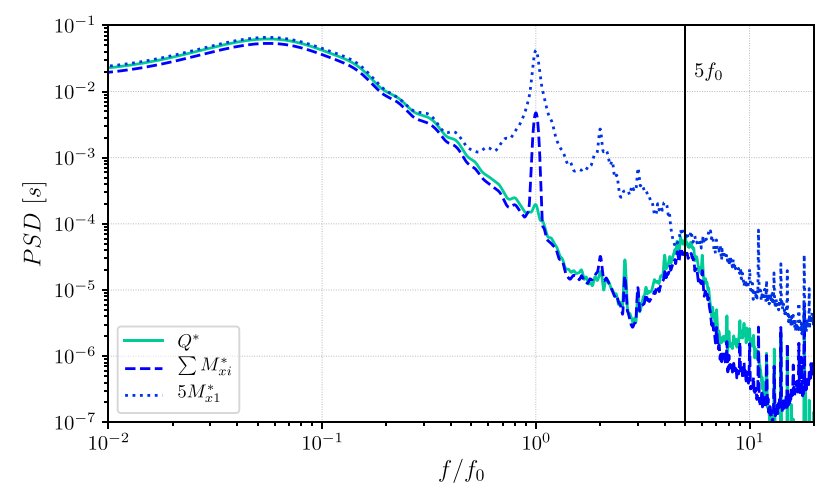

Fig. 32. Comparison of the power spectral densities of the rotor torque and the edgewise blade root bending moments obtained for the D12 turbine for TSR $=4.7$, $U_{\infty}=1.2 \mathrm{~m} / \mathrm{s}$ and the HTI case.

below $7 f_{0}$, except at $f_{0}$ where the peak is larger for $\sum M_{x i}^{*}$. The spectral densities of the thrust and the sum of the axial blade root forces (see Fig. 33) are almost identical.

The torque and thrust power spectral densities obtained at the nominal TSR operating point for the three $I_{\infty}$ cases are presented in Figs. 34 and 35. As for the ATIR results, the amplitudes of $S_{Q Q}$ and $S_{T T}$ increase with the turbulence intensity at low frequencies. For the LTI and MTI cases, more peaks are visible, particularly at $f_{0}$ and its harmonics.

The magnitude squared coherences obtained for the D12 turbine are presented in Fig. 36. The evolution of the different MSC are similar to those of the ATIR model, expect for a few points. For $\gamma_{Q u}^{2}$ and $\gamma_{T u}^{2}$, the cut-off frequency is slightly higher $(f \approx 2-3 \mathrm{~Hz})$. This may be due to

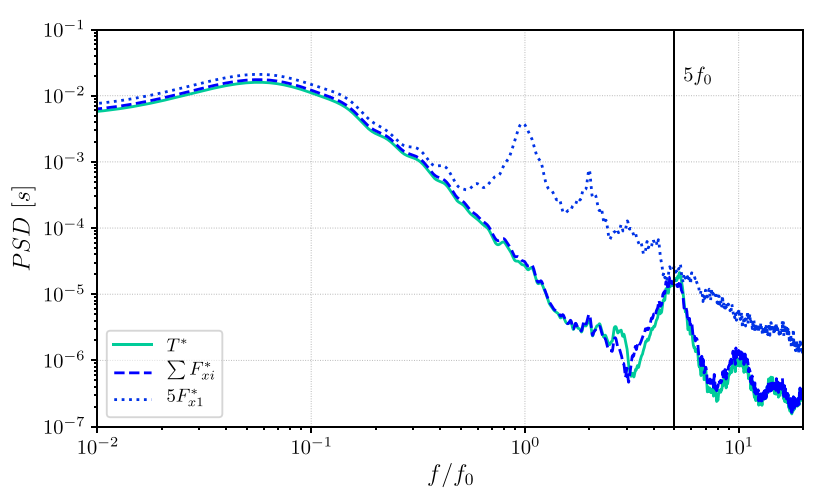

Fig. 33. Comparison of the power spectral densities of the rotor thrust and the axial blade root forces obtained for the D12 turbine for TSR $=4.7, U_{\infty}=1.2 \mathrm{~m} / \mathrm{s}$ and the HTI case.

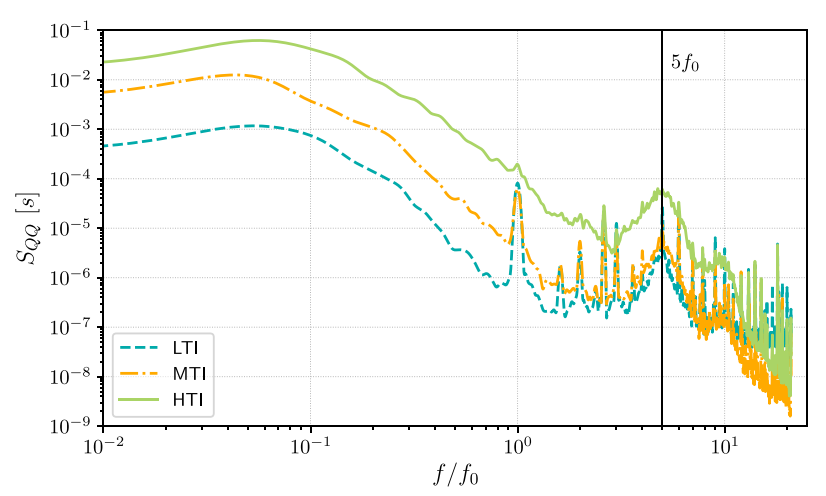

Fig. 34. Torque power spectral densities obtained for the D12 turbine for the three turbulence intensity cases at $U_{\infty}=1.2 \mathrm{~m} / \mathrm{s}$ and TSR $\approx 4.5$.

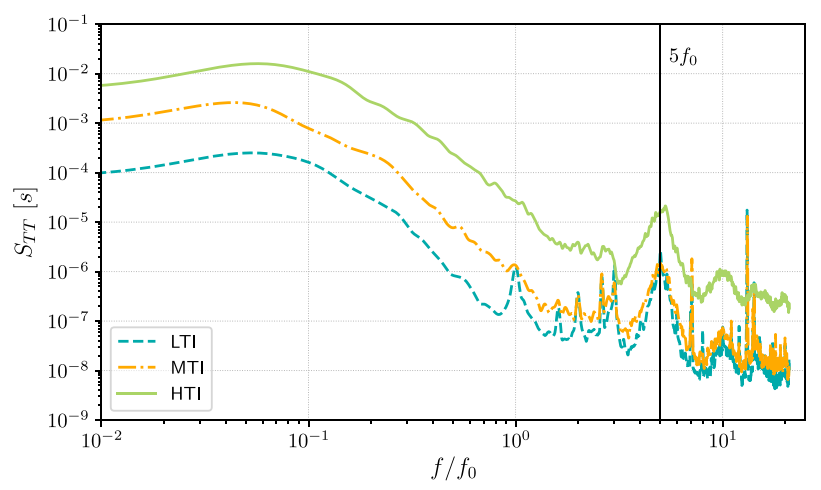

Fig. 35. Thrust power spectral densities obtained for the D12 turbine for the three turbulence intensity cases at $U_{\infty}=1.2 \mathrm{~m} / \mathrm{s}$ and TSR $\approx 4.5$.

the smaller diameter of the D12 model $(D=0.6 \mathrm{~m})$. At low frequencies, the coherences are surprisingly higher for both the MTI and LTI cases. This may be related to the fact that the characteristic length scale $l$ is similar for these two turbulence intensities (see Table 4), contrary to the ATIR turbine. However these values may not be accurate so further studies are required. Lastly, more fluctuations can be observed at high frequencies for $\gamma_{Q \omega}^{2}$ and $\gamma_{T \omega}^{2}$. This may be due to the blade supports (see Fig. 8) which create more disturbances.

\subsection{Comparison}

Fig. 37 show a comparison of the MSC obtained for the ATIR and D12 turbines with the ones computed for the IFREMER model, for two 

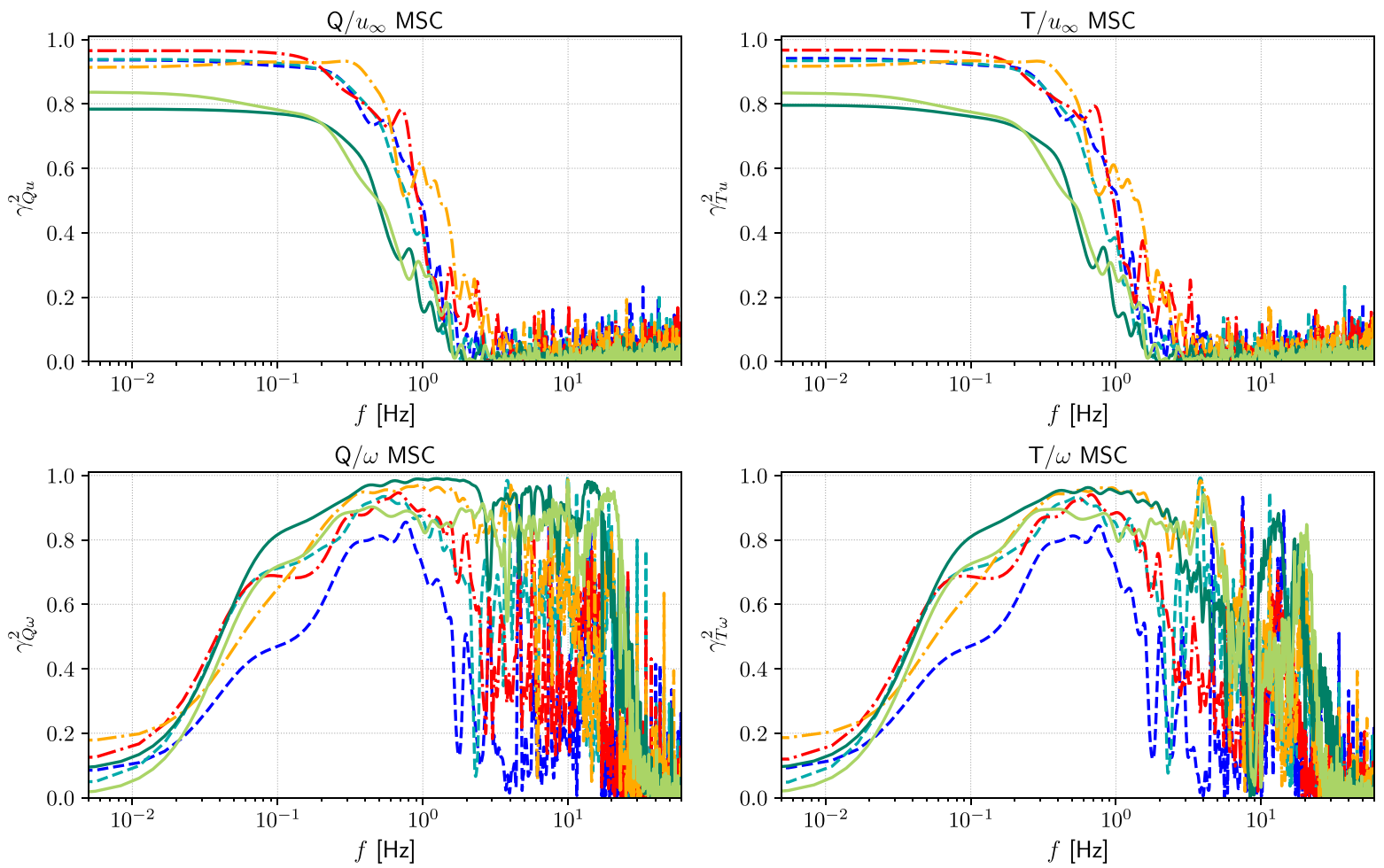

$\begin{array}{lll}---\mathrm{LTI}-\mathrm{TSR}=4.5 & ---\mathrm{MTI}-\mathrm{TSR}=4.5 & -\mathrm{HTI}-\mathrm{TSR}=4.5 \\ ---\mathrm{LTI}-\mathrm{TSR}=6 & ---\mathrm{MTI}-\mathrm{TSR}=6 & -\mathrm{HTI}-\mathrm{TSR}=6\end{array}$

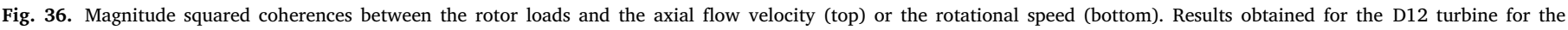
three turbulence intensity cases at $U_{\infty}=1.2 \mathrm{~m} / \mathrm{s}$ and two TSR values (TSR $\approx 4.5$ and TSR $\approx 6$ ).
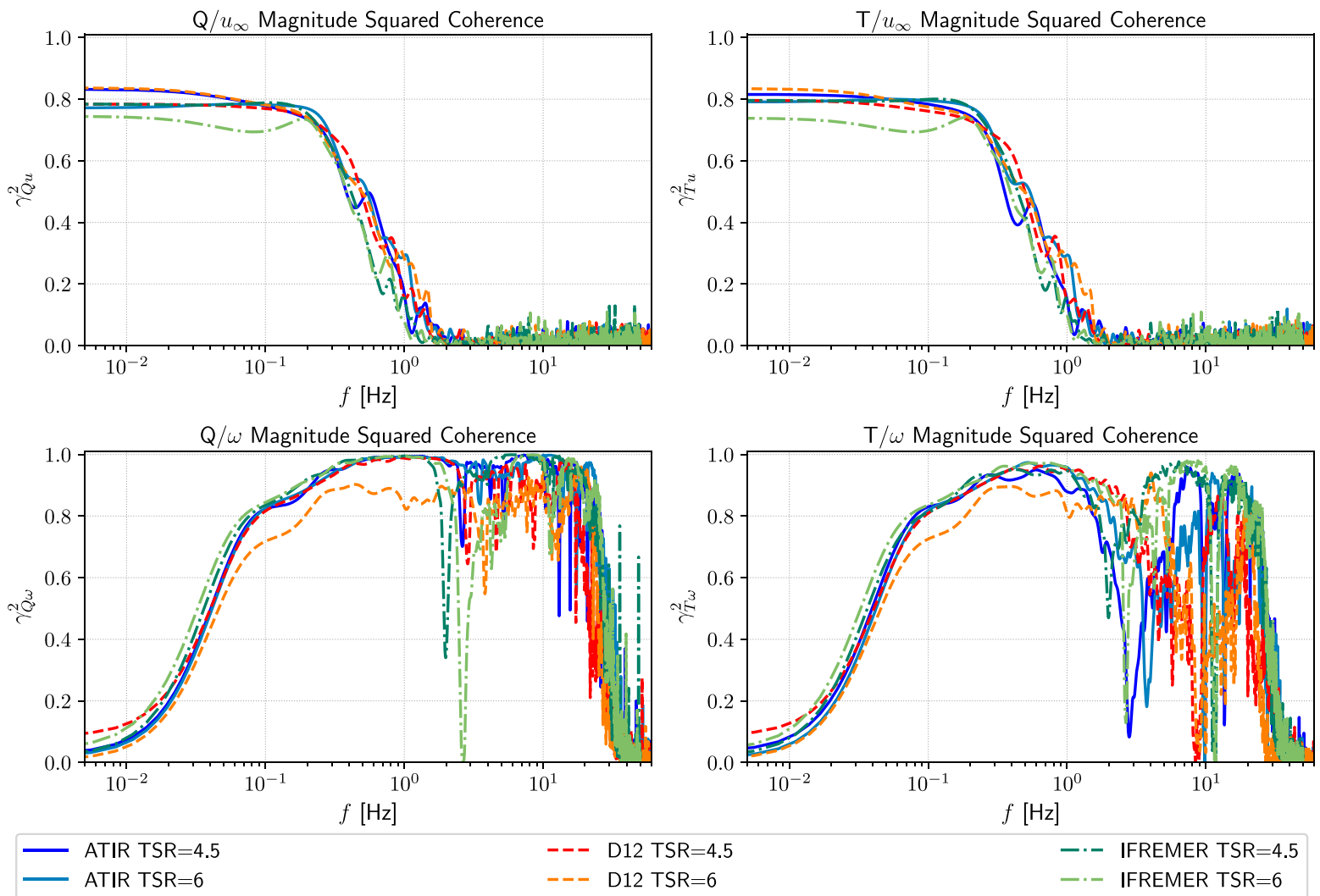

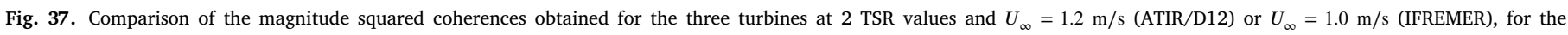
HTI case. 


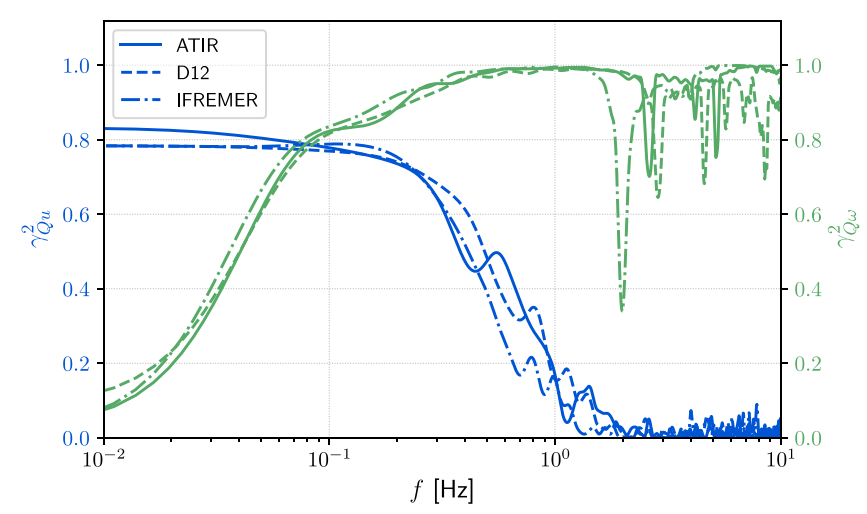

Fig. 38. Comparison of the magnitude squared coherences between the torque, the axial flow velocity and the rotational speed, obtained for the three turbines at TSR $\approx 4.5$ and $U_{\infty}=1.2 \mathrm{~m} / \mathrm{s}$ (ATIR/D12) or $U_{\infty}=1.0 \mathrm{~m} / \mathrm{s}$ (IFREMER), for the HTI case.

TSR values and the HTI condition. Since the speed control unit did not operate properly for the IFREMER turbine at $U_{\infty}=1.2 \mathrm{~m} / \mathrm{s}$ (the motor reached its power limit and there were large rotational speed variations), the coherences were calculated for $U_{\infty}=1.0 \mathrm{~m} / \mathrm{s}$. For the three turbines, the evolutions of the MSC are similar. For $\gamma_{Q u}^{2}$ and $\gamma_{T u}^{2}$, the cut-off frequency is slightly lower for the IFREMER model. This is consistent with the fact that its diameter is larger $(D=0.724 \mathrm{~m})$ and the flow velocity is smaller. For $\gamma_{O \omega}^{2}$ and $\gamma_{T \omega}^{2}$, the curves are almost superimposed at low frequencies (expect at TSR 6 for the D12 turbine). For $f \geq 1 \mathrm{~Hz}$, more discrepancies can be observed.

Lastly, a comparison of $\gamma_{Q u}^{2}$ and $\gamma_{Q \omega}^{2}$ for the three turbines is presented in Fig. 38. It can be observed that $\gamma_{Q \omega}^{2}$ becomes larger than $\gamma_{Q u}^{2}$ for $f \approx 0.08 \mathrm{~Hz}$ for all the models. Thus, at low frequencies the rotor load variations of the three turbines are mainly related to the flow velocity, whereas at high frequencies they are only related to the speed control unit.

\section{Conclusions}

During this study, scaled models of two industrial prototypes were tested: a 1:28 scale model of the 3-bladed horizontal axis turbine of Magallanes Renovables and a 1:20 scale model of the 5-bladed horizontal axis turbine of Sabella. Their performances were also compared to those of the open-geometry tidal turbine of the IFREMER. The results were divided by reference values in order to preserve the confidentiality of the industrial turbines. These laboratory trials were performed using three turbulence intensities, namely $I_{\infty} \approx 1.5 \%, I_{\infty} \approx 3 \%$ and $I_{\infty} \approx 15 \%$, and several upstream velocities without wave.

Synchronous bi-dimensional measurements of the flow velocity upstream of the turbine model were carried out. These measurements were used to characterise the ambient turbulence in the flume tank. Several quantities were computed, in particular: the turbulence intensity, the integral length scale, the dissipation rate of the turbulent kinetic energy $\varepsilon$ and other characteristic length scales. The estimation of $\varepsilon$ was done using the power spectral density of the flow velocity and Kolmogorov's $-5 / 3$ law. The results showed that the isotropy hypothesis is valid only for the case with the highest turbulence intensity $\left(I_{\infty} \approx 15 \%\right.$ ); this is easily explained by the grid and honeycomb used to regulate turbulence (see Section 2.2), which greatly restricts cross-stream lengthscales but has a smaller effect on along-stream lengthscales.

The analysis of the power and thrust coefficients was then presented. The averaged values and the standard deviations of these coefficients with respect to the tip speed ratio were provided. Among the three turbines tested, one turbine presented an important Reynolds effect for the lower tested velocities. Except from that, the anonymised power and thrust coefficients of these turbines converge for the different tested velocities. Different mean power $\left(C_{P}\right)$ and thrust $\left(C_{T}\right)$ curves are obtained for the different turbine designs, mainly in terms of operating range of TSR. It is important to remember here that the mean power and thrust values are anonymised for confidentiality issues. Increasing the ambient turbulence intensity tends to reduce the differences between the different rotors, especially between the scaled ATIR and D12 turbines. However, the turbulence rate has an important impact on the load fluctuations. Differences in solidity, blade profile and blade number do not seem to have such an influence on these fluctuating loads. Caution should be taken with this last conclusion as the three turbines are based on the same drive train. It may have a major influence partly hiding the differences due to the different rotor designs. In that respect, further analysis is still required to ascertain these individual conclusions.

Lastly, a spectral analysis of the rotor torque and thrust was carried out. Several peaks are identifiable in the load power spectral densities, in particular peaks due to the blades passage in front of the mast.
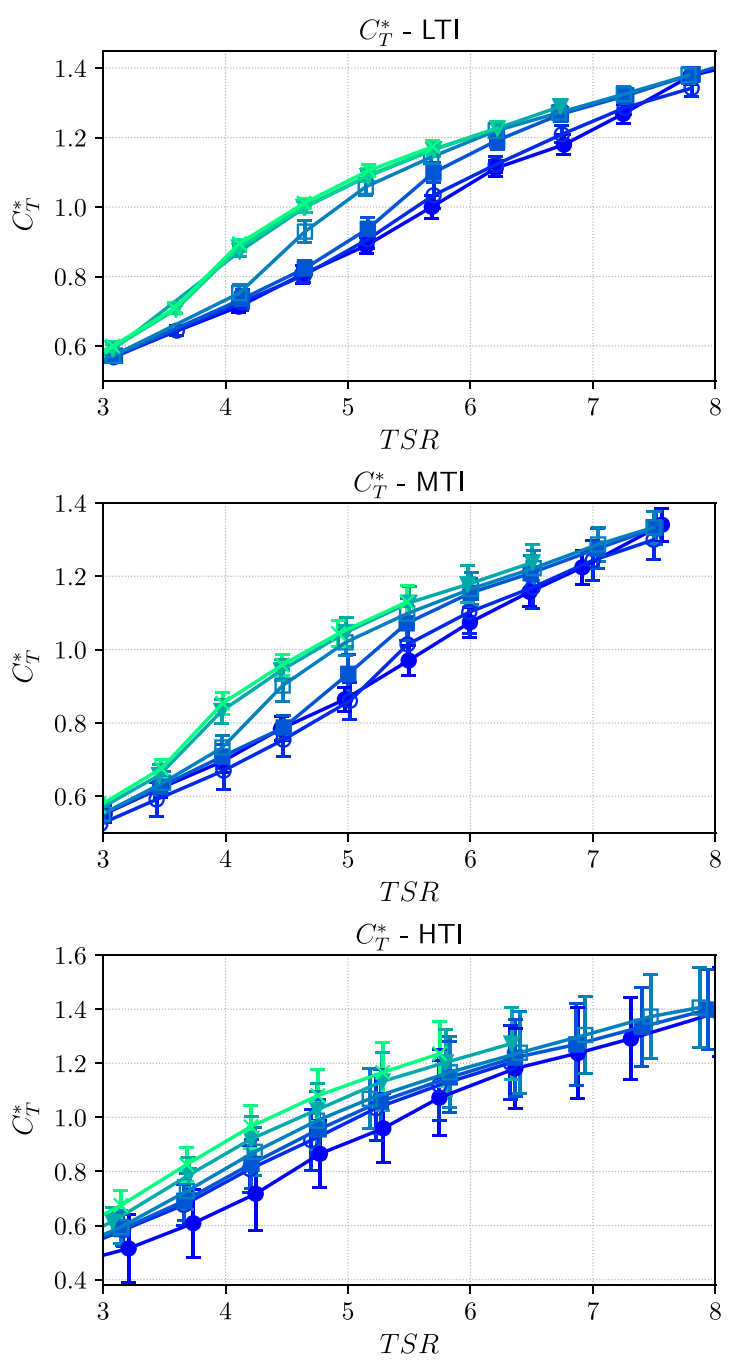

\begin{tabular}{|c|c|}
\hline 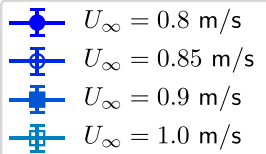 & $\begin{array}{l}\mp U_{\infty}=1.2 \mathrm{~m} / \mathrm{s} \\
\underset{I}{I} U_{\infty}=1.3 \mathrm{~m} / \mathrm{s} \\
\text { 平 } U_{\infty}=1.4 \mathrm{~m} / \mathrm{s}\end{array}$ \\
\hline
\end{tabular}

Fig. A.39. Mean thrust coefficients with the standard deviations, for the three ambient turbulence intensity cases, obtained for the ATIR turbine. The error bars denote \pm the standard deviation. 

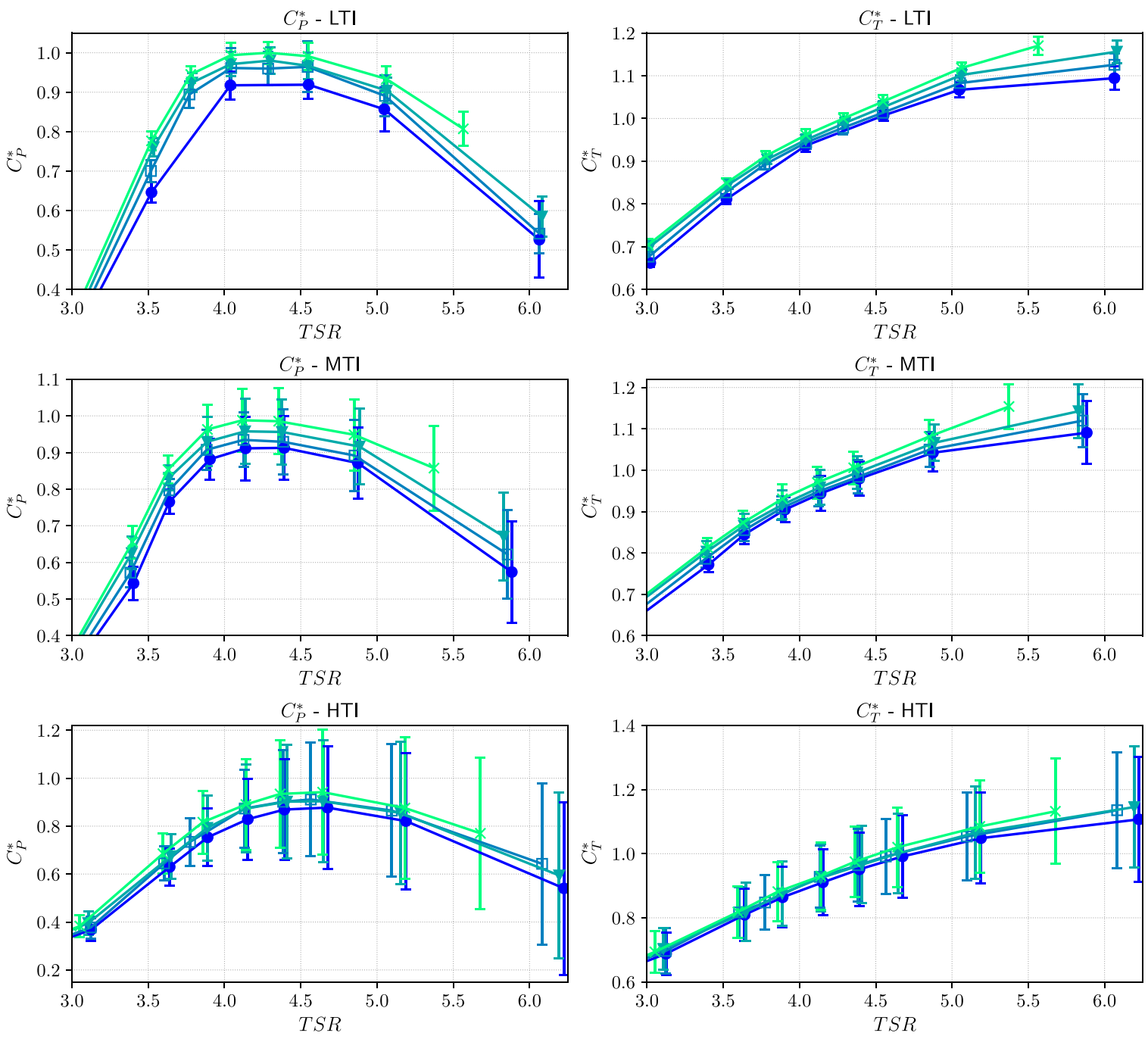

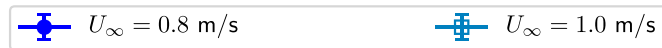

$$
\text { 平 } U_{\infty}=1.2 \mathrm{~m} / \mathrm{s} \quad \text { 平 } U_{\infty}=1.4 \mathrm{~m} / \mathrm{s}
$$

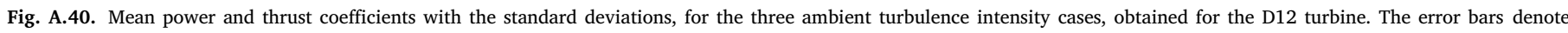
\pm the standard deviation.

The magnitude squared coherences, between the loads and the flow velocity and between the loads and the rotational speed, were also evaluated. The results showed that at low frequency the torque and thrust variations are related to the flow velocity whereas at high frequencies they are driven by the rotational speed control unit. For the highest turbulence intensity rate, the rotor type does not seem to impact the coherences between the rotor loads and the axial flow velocity. However, for the coherences between the rotor loads and the rotational speed, the rotor design has an influence for frequencies above $1 \mathrm{~Hz}$.

In future works, the influence of combined current and wave conditions will be studied. It will also be interesting to evaluate the impact of the drive train on the turbine performances.

\section{CRediT authorship contribution statement}

Myriam Slama: Formal analysis, Data curation, Writing - original draft, Visualization. Grégory Pinon: Conceptualization, Methodology, Writing - review \& editing, Supervision. Charifa El Hadi: Formal analysis, Investigation. Michael Togneri: Conceptualization, Writing review \& editing, Project administration, Funding acquisition. Benoît Gaurier: Methodology, Formal analysis, Investigation, Data curation. Grégory Germain: Conceptualization, Methodology, Writing - review \& editing. Jean-Valéry Facq: Methodology, Investigation. José Nuño:
Methodology, Resources. Pablo Mansilla: Methodology, Resources. Erwann Nicolas: Methodology, Resources. Julie Marcille: Methodology, Investigation, Resources. André Pacheco: Conceptualization, Project administration, Funding acquisition.

\section{Declaration of competing interest}

The authors declare that they have no known competing financial interests or personal relationships that could have appeared to influence the work reported in this paper.

\section{Acknowledgments}

This project is co-financed by the European Regional Development Fund (ERDF) through the Interreg Atlantic Area Programme, via the MONITOR project. This work was also supported in part by the ERDF and the Normandy Regional Council, France via programmes such as NEPTUNE, SEMARIN and DIADEMAR. This work benefits from studies carried out within the framework of H2020 MaRINET2 Round Robin tests and the Interreg 2 Seas MET-CERTIFIED project. This project was partly financially supported by the ERDF, the French government, IFREMER and the region Hauts-de-France in the framework of the project CPER 2015-2020 MARCO. 


\section{Appendix. Mean power and thrust coefficients with standard de- viations}

See Figs. A.39 and A.40.

\section{References}

Bahaj, A.S., Molland, A.F., Chaplin, J.R., Batten, W.M.J., 2007. Power and thrust measurements of marine current turbines under various hydrodynamic flow conditions in a cavitation tunnel and a towing tank. Renew. Energy 32 (3), 407-426. http://dx.doi.org/10.1016/j.renene.2007.10.001.

Blackmore, T., Myers, L.E., Bahaj, A.S., 2016. Effects of turbulence on tidal turbines: Implications to performance, blade loads, and condition monitoring. Int. J. Mar. Energy 14, 1-26. http://dx.doi.org/10.1016/j.ijome.2016.04.017, http:// www.sciencedirect.com/science/article/pii/S2214166916300297.

Diaz-Dorado, E., Carrillo, C., Cidrás, J., Román, D., Grande, J., 2021. Performance evaluation and modelling of the ATIR marine current turbine. IET Renewable Power Generation 15 (4), 821-838. http://dx.doi.org/10.1049/rpg2.12071.

Faudot, C., Dahlhaug, O.G., 2012. Prediction of wave loads on tidal turbine blades. Energy Procedia 20, 116-133. http://dx.doi.org/10.1016/j.egypro.2012. 03.014, Technoport 2012 - Sharing Possibilities and 2nd Renewable Energy Research Conference (RERC2012), http://www.sciencedirect.com/science/article/ pii/S1876610212007448.

Fernandez-Rodriguez, E., Stallard, T., Stansby, P., 2014. Experimental study of extreme thrust on a tidal stream rotor due to turbulent flow and with opposing waves. J. Fluids Struct. 51, 354-361. http://dx.doi.org/10.1016/j.jfluidstructs.2014.09.012, http://www.sciencedirect.com/science/article/pii/S0889974614002151.

Gaurier, B., Carlier, C., Germain, G., Pinon, G., Rivoalen, E., 2020. Three tidal turbines in interaction: An experimental study of turbulence intensity effects on wakes and turbine performance. Renew. Energy 148, 1150-1164. http://dx.doi. org/10.1016/j.renene.2019.10.006, http://www.sciencedirect.com/science/article/ pii/S0960148119314971.

Gaurier, B., Germain, G., Facq, J.-V., Bacchetti, T., 2018. Wave and current flume tank of IFREMER at boulogne-sur-mer. Description of the facility and its equipment. Report, FRANCE, http://dx.doi.org/10.13155/58163, https://archimer.ifremer.fr/ doc/00470/58163/.

Gaurier, B., Germain, G., Facq, J., Johnstone, C., Grant, A., Day, A., Nixon, E., Felice, F.D., Costanzo, M., 2015. Tidal energy "round robin" tests comparisons between towing tank and circulating tank results. Int. J. Mar. Energy 12, 87-109. http://dx.doi.org/10.1016/j.ijome.2015.05.005, Special Issue on Marine Renewables Infrastructure Network, http://www.sciencedirect.com/science/article/ pii/S2214166915000223.

Gaurier, B., Germain, G., Pinon, G., 2019. How to correctly measure turbulent upstream flow for marine current turbine performances evaluation? In: Soares, C.G. (Ed.), Advances in Renewable Energies Offshore: Proceedings of the 3rd International Conference on Renewable Energies Offshore (RENEW 2018), October 8-10, 2018, Lisbon, Portugal, 1st ed. Taylor \& Francis Group, pp. 23-30, ISBN 978-1-138-58535-5, Modelling Tidal Currents.

Guo, X., Yang, J., Gao, Z., Moan, T., Lu, H., 2018. The surface wave effects on the performance and the loading of a tidal turbine. Ocean Eng. 156, 120-134. http://dx.doi.org/10.1016/j.oceaneng.2018.02.033, http://www.sciencedirect.com/ science/article/pii/S0029801818301823.

Kaufmann, N., Carolus, T., Starzmann, R., 2019. Turbines for modular tidal current energy converters. Renew. Energy 142, 451-460. http://dx.doi.org/ 10.1016/j.renene.2019.04.120, http://www.sciencedirect.com/science/article/pii/ S0960148119306044.

Li, Y., Colby, J.A., Kelley, N., Thresher, R., Jonkman, B., Hughes, S., Inflow measurement in a tidal strait for deploying tidal current turbines: Lessons, opportunities and challenges, in: ASME 2010 29th International Conference on Ocean, Offshore and Arctic Engineering, 2010, pp. 569-576, http://dx.doi.org/10.1115/OMAE201020911.

Liu, P., Veitch, B., 2012. Design and optimization for strength and integrity of tidal turbine rotor blades. Energy 46 (1), 393-404. http://dx.doi.org/10.1016/j.energy. 2012.08.011, Energy and Exergy Modelling of Advance Energy Systems, http: //www.sciencedirect.com/science/article/pii/S0360544212006238.

Luznik, L., Flack, K.A., Lust, E.E., Taylor, K., 2013. The effect of surface waves on the performance characteristics of a model tidal turbine. Renew. Energy 58, 108-114. http://dx.doi.org/10.1016/j.renene.2013.02.022, http://www. sciencedirect.com/science/article/pii/S0960148113001316.
MacEnri, J., Reed, M., Thiringer, T., 2013. Influence of tidal parameters on seagen flicker performance. Phil. Trans. R. Soc. A 371 (1985), http://dx.doi.org/ 10.1098/rsta.2012.0247, arXiv:http://rsta.royalsocietypublishing.org/content/371/ 1985/20120247.full.pdf+html, http://rsta.royalsocietypublishing.org/content/371/ 1985/20120247.abstract.

Martinez, R., Payne, G.S., Bruce, T., 2018. The effects of oblique waves and currents on the loadings and performance of tidal turbines. Ocean Eng. 164, 55-64. http://dx. doi.org/10.1016/j.oceaneng.2018.05.057, https://www.sciencedirect.com/science/ article/pii/S0029801818309296.

McCaffrey, K., Fox-Kemper, B., Hamlington, P., Thomson, J., 2015. Characterization of turbulence anisotropy, coherence, and intermittency at a prospective tidal energy site: Observational data analysis. Renew. Energy 76, 441-453. http://dx.doi.org/ 10.1016/j.renene.2014.11.063.

Medina, O.D., Schmitt, F.G., Calif, R., Germain, G., Gaurier, B., 2017. Turbulence analysis and multiscale correlations between synchronized flow velocity and marine turbine power production. Renew. Energy 112, 314-327. http://dx.doi. org/10.1016/j.renene.2017.05.024, http://www.sciencedirect.com/science/article/ pii/S0960148117304093.

Milne, I., Day, A., Sharma, R., Flay, R., 2016. The characterisation of the hydrodynamic loads on tidal turbines due to turbulence. Renew. Sustain. Energy Rev. 56, 851864. http://dx.doi.org/10.1016/j.rser.2015.11.095, http://www.sciencedirect.com/ science/article/pii/S1364032115013623.

Milne, I.A., Sharma, R.N., Flay, R.G.J., Bickerton, S., 2013. Characteristics of the turbulence in the flow at a tidal stream power site. Phil. Trans. R. Soc. A 371 (1985), http://dx.doi.org/10.1098/rsta.2012.0196, arXiv:http://rsta.royalsocietypublishing. org/content/371/1985/20120196.full.pdf+html, http://rsta.royalsocietypublishing. org/content/371/1985/20120196.abstract.

Murray, R.E., Ordonez-Sanchez, S., Porter, K.E., Doman, D.A., Pegg, M.J., Johnstone, C.M., 2018. Towing tank testing of passively adaptive composite tidal turbine blades and comparison to design tool. Renew. Energy 116, 202-214. http://dx.doi. org/10.1016/j.renene.2017.09.062, http://www.sciencedirect.com/science/article/ pii/S0960148117309254.

Mycek, P., Gaurier, B., Germain, G., Pinon, G., Rivoalen, E., 2014. Experimental study of the turbulence intensity effects on marine current turbines behaviour. Part I: One single turbine. Renew. Energy 66, 729-746. http://dx.doi. org/10.1016/j.renene.2013.12.036, http://www.sciencedirect.com/science/article/ pii/S096014811400007X.

Payne, G.S., Stallard, T., Martinez, R., Bruce, T., 2018. Variation of loads on a three-bladed horizontal axis tidal turbine with frequency and blade position. J. Fluids Struct. 83, 156-170. http://dx.doi.org/10.1016/j.jfluidstructs.2018.08.010, http://www.sciencedirect.com/science/article/pii/S0889974617306734.

Pinon, G., Hurst, M.F., Lukeba, E., 2017. Semi-analytical estimate of energy production from a tidal turbine farm with the account of ambient turbulence. Int. J. Mar. Energy 19, 70-82. http://dx.doi.org/10.1016/j.ijome.2017.05.003, http://www. sciencedirect.com/science/article/pii/S2214166917300516.

Pope, S.B., 2000. Turbulent Flows. Cambridge University Press, Cambridge, http:// dx.doi.org/10.1017/cbo9780511840531, https://www.cambridge.org/core/books/ turbulent-flows/C58EFF59AF9B81AE6CFAC9ED16486B3A.

Saddoughi, S.G., Veeravalli, S.V., 1994. Local isotropy in turbulent boundary layers at high Reynolds number. J. Fluid Mech. 268, 333-372. http: //dx.doi.org/10.1017/s0022112094001370, https://www.cambridge.org/ core/article/local-isotropy-in-turbulent-boundary-layers-at-high-reynoldsnumber/1D77E82B20BF159F676C7871C1379578.

Scheijgrond, P., Southall, A., Bittencourt, C., Davies, P., Mathys, P., Germain, G., Geertzen, M., 2019. Advencing IEC standardization and certification for tidal energy converters. In: Vicinanza, D. (Ed.), Proceedings of the Thirteenth European Wave and Tidal Energy Conference. EWTEC, Universitá degli Studi della Campania "Luigi Vanvitelli", Italy, ISSN: 2309-1983.

Stoica, P., Moses, R.L., 2005. Spectral Analysis of Signals. Prentice Hall,

Thomson, J., Polagye, B., Durgesh, V., Richmond, M., 2012. Measurements of turbulence at two tidal energy sites in puget sound, WA. IEEE J. Ocean. Eng. 37 (3), 363-374. http://dx.doi.org/10.1109/JOE.2012.2191656.

Togneri, M., Buck, E., MacLeod, A., Nicolas, E., Nuño, J., O'Connor, M., Pacheco, A., Pinon, G., Masters, I., 2019. Multi-model analysis of tidal turbine reliability. In: Vicinanza, D. (Ed.), Proceedings of the Thirteenth European Wave and Tidal Energy Conference. EWTEC, Universitá degli Studi della Campania "Luigi Vanvitelli", Italy, ISSN: 2309-1983.

Togneri, M., Lewis, M., Neill, S., Masters, I., 2017. Comparison of ADCP observations and 3D model simulations of turbulence at a tidal energy site. Renew. Energy 114, 273-282. http://dx.doi.org/10.1016/j.renene.2017.03.061, http:// www.sciencedirect.com/science/article/pii/S0960148117302537, Wave and Tidal Resource Characterization.

Togneri, M., Pinon, G., Carlier, C., Bex, C.C., Masters, I., 2020. Comparison of synthetic turbulence approaches for blade element momentum theory prediction of tidal turbine performance and loads. Renew. Energy 145, 408-418. http://dx.doi. org/10.1016/j.renene.2019.05.110, http://www.sciencedirect.com/science/article/ pii/S0960148119307839. 